$\mathrm{PSU} / \mathrm{TH} / 172$

February 1997

\title{
Real Forms of Nonlinear Superconformal and Quasi-Superconformal Algebras and Their Unified Realization
}

\author{
Behzad Bina and Murat Günaydin 耳 \\ Department of Physics \\ 104 Davey Lab. \\ Penn State University \\ University Park, PA 16802
}

\begin{abstract}
We give a complete classification of the real forms of simple nonlinear superconformal algebras (SCA) and quasi-superconformal algebras (QSCA) and present a unified realization of these algebras with simple symmetry groups. This classification is achieved by establishing a correspondence between simple nonlinear QSCA's and SCA's and quaternionic and superquaternionic symmetric spaces of simple Lie groups and Lie supergroups, respectively. The unified realization we present involves a dimension zero scalar field (dilaton), dimension-1 symmetry currents, and dimension-1/2 free bosons for QSCA's and dimension-1/2 free fermions for SCA's. The free bosons and fermions are associated with the quaternionic and superquaternionic symmetric spaces of corresponding Lie groups and Lie supergroups, respectively. We conclude with a discussion of possible applications of our results.
\end{abstract}

\footnotetext{
${ }^{1}$ Work supported in part by the National Science Foundation under Grant Number PHY-9631332.

e-mail: murat@phys.psu.edu
} 


\section{Introduction}

The infinite dimensional conformal group in two dimensions $(d=2)$ plays a fundamental role in the formulation of string theories [1] and in the understanding of critical phenomena in two dimensional physical systems. Supersymmetric extensions of the conformal group underlie superstring theories. The spacetime supersymmetric perturbative vacua of superstrings are described by extended superconformal field theories. The extended superconformal algebras also have applications to integrable systems and to topological field theories.

Conformal group is finite dimensional in higher than two dimensions $(d>2)$. For a d-dimensional space with Lorentzian signature, the conformal group is $S O(d, 2)(d>2)$. The "global" subgroup $S O(2,2)$ of the infinite dimensional conformal group in two dimensions with Lorentzian signature is not simple and decomposes as

$$
S O(2,2) \cong S O(2,1) \times S O(2,1)
$$

where the two $S O(2,1)$ factors act on the light-cone coordinates $x^{+}=\tau+\sigma$ and $x^{-}=\tau-\sigma$ (left and right movers), respectively. This fact allows one to have different numbers of supersymmetries in the left and right moving sectors. Using the known classification of simple Lie superalgebras [2], a complete classification of the supersymmetric extensions of the global conformal group $S O(2,2)$ in two dimensions was given in [3]. The finite dimensional Lie superalgebras of the global superconformal groups do not always admit extensions to infinite-dimensional linear superconformal algebras (SCA's) with generators of non-negative conformal dimensions. Such linear infinite dimensional extensions exist only when the number $N$ of supersymmetries is less than or equal to four 田, 5, 6, 开. Known SCA's with $N>4$ and generators of non-negative conformal dimensions are either of the quadratically nonlinear type [8, 9] or are soft algebras [10, 11, 12] with field-dependent structure "constants." The $N$-extended quadratically nonlinear SCA's that were originally introduced by Knizhnik and Bershadsky [8, 9] involve generators of conformal dimensions $2, \frac{3}{2}$, and 1 only.

The reductive quadratically nonlinear SCA's with compact symmetry groups were classified in [13, 14]. These "reductive" nonlinear SCA's linearize in the limit of infinite central charge, and, in this limit, have finite dimensional global superconformal algebras as subalgebras. As a consequence, the classification of the reductive nonlinear SCA's with compact 
symmetry groups, given in [13, 14, follows directly from the list of finite dimensional global SCA's [3].

The nonlinear SCA's of the type introduced by Knizhnik and Bershadsky satisfy the usual spin and statistics connection and they can all be obtained via the Drinfeld-Sokolov type quantum Hamiltonian reduction of affine Lie superalgebras [15]. The realizations of the nonlinear SCA's with $U(n)$ and $S O(n)$ symmetry were studied in [16, 17] and those of the exceptional $N=8$ and $N=7$ SCA's in [18]. The quantum Hamiltonian reduction can also be used to obtain $W_{N}$ algebras [19] of the type first introduced by Zamolodchikov [20].

As was pointed out by Polyakov [21], there are two distinct reductions of affine $S L(3, R)$ algebra. One leads to the $W_{3}$ algebra of Zamolodchikov and the second leads to a nonlinear SCA, $W_{3}^{2}$, with two bosonic supersymmetry generators of conformal dimension $\frac{3}{2}$. Bershadsky developed the systematics of this type of algebras and studied the case of $W_{3}^{2}$ in detail [22]. These results were generalized greatly by Romans who introduced two infinite families of nonlinear SCA's with bosonic supersymmetry generators and called them quasi-superconformal algebras (QSCA's) [23]. A classification of the complex forms of reductive QSCA's was given in [13]. In 24], nonlinear SCA's with both fermionic and bosonic supersymmetry generators were studied. We shall refer to such nonlinear SCA's with both bosonic and fermionic supersymmetry generators as super-quasi-superconformal algebras (SQSCA).

In 25], the classical and quantum BRST operators for nonlinear SCA's were studied. These results were generalized to QSCA's and SQSCA's in [26.

In this paper, we will study nonlinear SCA's and QSCA's in full generality. In Section 2, we give the operator product expansions as well as the (super-)commutation relations of nonlinear SCA's and QSCA's and study the constraints imposed by the (super-)Jacobi identities. In Section 3, we establish a one-to-one correspondence between quaternionic symmetric spaces of simple Lie groups and simple nonlinear QSCA's. Using this correspondence, we give a complete classification of the real forms of simple nonlinear QSCA's in Section 4. In Section 5, we establish a one-to-one correspondence between "super-quaternionic" symmetric spaces of simple Lie supergroups and simple nonlinear SCA's and give a complete classification of the real forms of these algebras. In Section 6, we give a unified realization of nonlinear SCA's and QSCA's with simple symmetry groups in terms of a free scalar field (dilaton), dimension-1 currents that generate an affine symme- 
try algebra, and free dimension- $1 / 2$ fermions and bosons corresponding to the super-quaternionic and quaternionic symmetric spaces, respectively. By realizing these affine symmetry currents in terms of free bosons, one can obtain a unified realization of these algebras in terms of free fields alone. In our unified realization, we recover as special cases the realization of $S O(n)$ SCA's of [16] and $S p(2 n)$ QSCA's of [23]. For the $S O(n)$ and $S p(2 n)$ algebras, the realization is considerably simpler, since, for these algebras, the term in the supercurrents that are trilinear in the dimension- $1 / 2$ fields drop out. We should stress also that, to our knowledge, classification of different real forms of nonlinear algebras has not appeared in the literature before. In fact, in some of the literature, there seems to be an implicit assumption that symmetry groups of these algebras can always be chosen to be compact. For nonlinear SCA's, there always exists a real form for which the symmetry group is compact. However, for QSCA's, this is, in general, not the case.

We conclude with a discussion of some of the possible applications of our results.

\section{Nonlinear Quasi-Superconformal and Supercon- formal Algebras}

QSCA's (SCA's) involve Virasoro or energy-momentum generators of conformal dimension 2, bosonic (fermionic) supersymmetry generators of dimension $\frac{3}{2}$, and symmetry currents of dimension 1 . The defining relations of these algebras take on their simplest form when written in terms of operator products. Let $T(z)$ denote the energy-momentum tensor, $G^{\alpha}(z)$ be the bosonic (fermionic) supersymmetry generators, and $J^{a}(z)$ be the currents of the affine symmetry algebra $\hat{g}$. Their OPE's read as [13, 26] ${ }^{2}$

$$
\begin{aligned}
T(z) T(w) & =\frac{c / 2}{(z-w)^{4}}+\frac{2 T(w)}{(z-w)^{2}}+\frac{\partial T(w)}{(z-w)}+\cdots \\
T(z) G^{\alpha}(w) & =\frac{\frac{3}{2} G^{\alpha}(w)}{(z-w)^{2}}+\frac{\partial G^{\alpha}(w)}{(z-w)}+\cdots \\
T(z) J^{a}(w) & =\frac{J^{a}(w)}{(z-w)^{2}}+\frac{\partial J^{a}(w)}{(z-w)}+\cdots \\
G^{\alpha}(z) G^{\beta}(w) & =\frac{b \Omega^{\alpha \beta}}{(z-w)^{3}}+\frac{\sigma \lambda_{a}^{\alpha \beta} J^{a}(w)}{(z-w)^{2}}+\frac{\frac{1}{2} \sigma \lambda_{a}^{\alpha \beta} \partial J^{a}(w)}{(z-w)}
\end{aligned}
$$

\footnotetext{
${ }^{2}$ Our notations follow those of [13] closely.
} 


$$
\begin{aligned}
& +\frac{2 \Omega^{\alpha \beta} T(w)}{(z-w)}+\frac{\gamma P_{a b}^{\alpha \beta}: J^{a} J^{b}:(w)}{(z-w)}+\cdots \\
J^{a}(z) G^{\alpha}(w)= & \frac{-\lambda^{a, \alpha}{ }_{\beta} G^{\beta}(w)}{(z-w)}+\cdots \\
J^{a}(z) J^{b}(w) & =\frac{-\frac{1}{2} k \ell^{2} \eta^{a b}}{(z-w)^{2}}+\frac{f_{c}^{a b} J^{c}(w)}{(z-w)}+\cdots,
\end{aligned}
$$

where $\lambda^{a}(a, b, \ldots=1, \ldots, D=\operatorname{dimg})$ are the representation $\rho$ matrices of $g$ under which the supersymmetry generators $G^{\alpha}$ transform. The representation $\rho$ is symplectic for QSCA's and orthogonal for SCA's, and the matrix elements $\lambda_{\alpha \beta}^{a}(\alpha, \beta, \ldots=1, \ldots, N=\operatorname{dim} \rho)$ of $\lambda$ 's have the symmetry property

$$
\lambda_{\alpha \beta}^{a}=\epsilon \lambda_{\beta \alpha}^{a},
$$

where $\epsilon=+1$ for QSCA's and $\epsilon=-1$ for SCA's. The supersymmetry indices $\alpha, \beta, \ldots$ will be raised and lowered with the help of a $g$-invariant tensor ("metric" in the representation space $\rho$ ) $\Omega^{\alpha \beta}=-\epsilon \Omega^{\beta \alpha}$ and its inverse $\Omega_{\alpha \beta}$

$$
\begin{aligned}
& V^{\alpha} \equiv V_{\beta} \Omega^{\alpha \beta} \\
& V_{\alpha} \equiv \Omega_{\beta \alpha} V^{\beta} \\
& \Omega^{\alpha \beta} \Omega_{\gamma \beta}=\delta^{\alpha}{ }_{\gamma} .
\end{aligned}
$$

Note that $V^{\alpha} W_{\alpha}=-\epsilon V_{\alpha} W^{\alpha}$. We shall raise and lower the adjoint indices $a, b, \ldots$ of $g$ by using the tensor $\eta^{a b}=\eta^{b a}$ and its inverse $\eta_{a b}$ that is related to the Cartan-Killing metric $g_{a b}$ of $g$ via

$$
g_{a b}=f_{a c d} f_{b}{ }^{d c}=-C_{a d j} \eta_{a b} .
$$

where $f_{a b}^{c}$ 's are the structure constants of $g$, and $C_{a d j}$ is the eigenvalue of the second order Casimir operator in the adjoint representation. We should note that in 13, 26], where complex forms of the Lie algebra $g$ were studied, $\eta_{a b}$ was taken to be $\delta_{a b}$. For real forms of nonlinear QSCA's, $\eta_{a b}$ can not be taken to be $\delta_{a b}$ in general, as will be shown below. The $\lambda^{a}$ 's satisfy the commutation relations

$$
\lambda_{a \beta}^{\alpha} \lambda_{b \gamma}^{\beta}-\lambda_{b \beta}^{\alpha} \lambda_{a \gamma}^{\beta}=f_{a b}^{c} \lambda_{c \gamma}^{\alpha},
$$


and are normalized such that for simple $g$ we have

$$
\begin{aligned}
& \lambda_{\alpha \beta}^{a} \lambda^{b, \beta \alpha}=-i_{\rho} \ell^{2} \eta^{a b} \\
& \lambda_{\alpha \beta}^{a} \lambda_{a}^{\beta \gamma}=-C_{\rho} \delta_{\alpha}^{\gamma},
\end{aligned}
$$

where $i_{\rho}$ and $C_{\rho}$ are the Dynkin index and the eigenvalue of the second order Casimir operator of the representation $\rho$, respectively. They are related as

$$
i_{\rho}=\frac{N C_{\rho}}{D \ell^{2}} .
$$

The length squared, $\ell^{2}$, of the longest root is normalized such that it is 2 for the simply-laced Lie algebras, 4 for $B_{n}, C_{n}$, and $F_{4}$, and 6 for $G_{2}$. The dual Coxeter number $\check{g}$ of a simple Lie algebra $g$ is related to the eigenvalue $C_{a d j}$ of the second order Casimir in the adjoint representation by

$$
C_{a d j}=-\epsilon \ell^{2} \check{g}
$$

As was shown in [13], the necessary and sufficient condition for the existence of nonlinear QSCA's or SCA's is that the $\lambda$ matrices satisfy the identity

$$
\lambda^{a, \alpha \beta} \lambda_{a \delta}^{\gamma}-\lambda^{a, \gamma \alpha} \lambda_{a \delta}^{\beta}=\frac{2}{\sigma_{0}}\left(\Omega^{\alpha \beta} \delta_{\delta}^{\gamma}-2 \Omega^{\beta \gamma} \delta_{\delta}^{\alpha}+\Omega^{\gamma \alpha} \delta_{\delta}^{\beta}\right),
$$

where, for simple $g$ and irreducible $\rho, \sigma_{0}$ is given by

$$
\sigma_{0}=\frac{-2(1+\epsilon N)}{C_{\rho}}
$$

The constants $c, b, \sigma$, and $\gamma$ are determined by imposing the (super-) Jacobi identities. For this, we need the OPE's of the generators $T(z), G^{\alpha}(z)$, and $J^{a}(z)$ with current bilinears. These are given by ${ }^{3}$

$$
\begin{aligned}
T(z): J^{a} J^{b}:(w)= & -\frac{1}{2} \frac{k \ell^{2} \eta^{a b}}{(z-w)^{4}}+\frac{f_{c}^{a b} J^{c}(w)}{(z-w)^{3}}+\frac{2: J^{a} J^{b}:(w)}{(z-w)^{2}} \\
& +\frac{\partial: J^{a} J^{b}:(w)}{(z-w)}+\cdots \\
G^{\alpha}(z): J^{a} J^{b}:(w)= & \frac{1}{(z-w)^{2}} \lambda_{\beta}^{a, \alpha} \lambda_{\gamma}^{b, \beta} G^{\gamma}(w)
\end{aligned}
$$

\footnotetext{
${ }^{3}$ All the symmetrizations and antisymmetrizations in this paper are defined with unit weight.
} 


$$
\begin{aligned}
& -\frac{1}{(z-w)} \lambda_{\beta}^{b, \alpha} \lambda_{\gamma}^{a, \beta} \partial G^{\gamma}(w) \\
& +\frac{1}{(z-w)}: G^{\beta} J^{(a}:(w) \lambda^{b), \alpha}{ }_{\beta}+\cdots \\
J^{c}(z): J^{a} J^{b}:(w)= & -\frac{1}{2} \frac{k \ell^{2} f^{c a b}}{(z-w)^{3}}-\frac{1}{2} \frac{k \ell^{2}}{(z-w)^{2}} \eta^{c(a} J^{b)}(w) \\
& +\frac{1}{(z-w)^{2}} f_{d}^{c a} f_{e}^{d b} J^{e}(w) \\
& +\frac{1}{(z-w)} f_{d}^{c a}: J^{d} J^{b}:(w) \\
& +\frac{1}{(z-w)} f_{d}^{c b}: J^{a} J^{d}:(w)+\cdots
\end{aligned}
$$

In terms of the modes, the OPE's (2-1) and (2-12) read as

$$
\begin{aligned}
{\left[L_{m}, L_{n}\right]=} & (m-n) L_{m+n}+\frac{1}{12} c m\left(m^{2}-1\right) \delta_{m+n, 0} \\
{\left[L_{m}, G_{r}^{\alpha}\right]=} & \left(\frac{1}{2} m-r\right) G_{m+r}^{\alpha} \\
{\left[L_{m}, J_{n}^{a}\right]=} & -n J_{m+n}^{a} \\
{\left[G_{r}^{\alpha}, G_{s}^{\beta}\right\}=} & \frac{1}{2} b\left(r^{2}-\frac{1}{4}\right) \Omega^{\alpha \beta} \delta_{r+s, 0}+\frac{1}{2} \sigma(r-s) \lambda_{a}^{\alpha \beta} J_{r+s}^{a} \\
& +2 \Omega^{\alpha \beta} L_{r+s}+\gamma P_{a b}^{\alpha \beta}\left(J^{a} J^{b}\right)_{r+s} \\
{\left[J_{m}^{a}, G_{r}^{\alpha}\right]=} & -\lambda^{a, \alpha}{ }_{\beta} G_{m+r}^{\beta} \\
{\left[J_{m}^{a}, J_{n}^{b}\right]=} & -\frac{1}{2} k \ell^{2} \eta^{a b} m \delta_{m+n, 0}+f^{a b}{ }_{c} J_{m+n}^{c} \\
{\left[L_{m},\left(J^{a} J^{b}\right)_{n}\right]=} & -\frac{1}{12} k \ell^{2} \eta^{a b} m\left(m^{2}-1\right) \delta_{m+n, 0}+\frac{1}{2} m(m+1) f_{c}^{a b} J_{m+n}^{c} \\
& +(m-n)\left(J^{a} J^{b}\right)_{m+n} \\
{\left[G_{r}^{\alpha},\left(J^{a} J^{b}\right)_{n}\right]=} & \left(r+\frac{1}{2}\right) \lambda^{a, \alpha}{ }_{\beta} \lambda^{b, \beta}{ }_{\gamma} G_{r+n}^{\gamma}+\left(r+n+\frac{3}{2}\right) \lambda^{b, \alpha}{ }_{\beta} \lambda^{a, \beta}{ }_{\gamma} G_{r+n}^{\gamma} \\
& +\left(G^{\beta} J^{(a}\right)_{r+n} \lambda^{b), \alpha}{ }_{\beta} \\
{\left[J_{m}^{c},\left(J^{a} J^{b}\right)_{n}\right]=} & -\frac{1}{12} k \ell^{2} m \eta^{c(a} J_{m+n}^{b)}-\frac{1}{4} k \ell^{2} f^{c a b} m(m-1) \delta_{m+n, 0} \\
& +f^{c a d} f_{d}^{b} e_{m}{ }_{m} J_{m+n}{ }_{m+n}+f_{d}^{c a}\left(J^{d} J^{b}\right)_{m+n} \\
& +f^{c b}{ }_{d}\left(J^{a} J^{d}\right)_{m+n} .
\end{aligned}
$$

The bracket $[.$, . $\}$ denotes a commutator for QSCA's $(\epsilon=+1)$ and an anticommutator for SCA's $(\epsilon=-1)$. We verified that all the Jacobi 
(super-Jacobi) conditions are satisfied if the following relations hold:

$$
\begin{aligned}
& b=-\frac{1}{2} \epsilon \sigma k \ell^{2} \\
& P_{a b}^{\alpha \beta} \lambda_{\delta}^{a, \gamma}+P_{a b}^{\gamma \alpha} \lambda_{\delta}^{a, \beta}+P_{a b}^{\beta \gamma} \lambda_{\delta}^{a, \alpha}=0 \\
& \left(b-\frac{2}{3} c\right) \Omega^{\alpha \beta}+\frac{1}{3} \gamma k \ell^{2} P_{a b}^{\alpha \beta} \eta^{a b}=0 \\
& P_{c f}^{\alpha \beta} f_{e}^{a c}+P_{e b}^{\alpha \beta} f_{f}^{a b}+P_{e f}^{\gamma \beta} \lambda^{a, \alpha}{ }_{\gamma}-\epsilon P_{e f}^{\gamma \alpha} \lambda^{a, \beta}{ }_{\gamma}=0 \\
& 2 \Omega^{\alpha \beta} \delta_{e}^{a}-\frac{\sigma}{2}\left(\epsilon \lambda^{a, \beta}{ }_{\gamma} \lambda_{e}^{\gamma \alpha}-\lambda^{a, \alpha}{ }_{\gamma} \lambda_{e}^{\gamma \beta}\right)-k \ell^{2} \gamma P_{b e}^{\alpha \beta} \eta^{a b}= \\
& -\gamma P_{c b}^{\alpha \beta} f_{d}^{a c} f_{e}^{d b}(2-18) \\
& \frac{-\sigma}{2}\left(\lambda^{a, \alpha \beta} \lambda_{a \delta}^{\gamma}-\lambda^{a, \gamma \alpha} \lambda_{a \delta}^{\beta}\right)+\Omega^{\alpha \beta} \delta_{\delta}^{\gamma}-2 \Omega^{\beta \gamma} \delta_{\delta}^{\alpha}+\Omega^{\gamma \alpha} \delta_{\delta}^{\beta}= \\
& \gamma P_{a b}^{\beta \gamma} \lambda^{a, \alpha}{ }_{\mu} \lambda^{b, \mu}{ }_{\delta} \cdot(2-19)
\end{aligned}
$$

Before proceeding to solve the conditions $(2-14)-(2-19)$, we note a special identity. In the solution of these conditions, we have to evaluate the expression $\lambda_{a}{ }_{\beta}{ }_{\beta} \lambda^{b, \beta}{ }_{\gamma} \lambda^{a, \gamma}{ }_{\delta}$, which can be done in two different ways. The first is to use the commutation relations to switch the order of the first two (or the last two) matrices. Doing so, we get 1

$$
\lambda_{a \beta}^{\alpha} \lambda_{\gamma}^{b, \beta} \lambda_{\delta}^{a, \gamma}=\left(\epsilon C_{\rho}+\frac{1}{2} C_{a d j}\right) \lambda_{\delta}^{b, \alpha} .
$$

The second way is to use $(2-10)$ to exchange the $\alpha$ and $\gamma$ indices. This way, we find

$$
\lambda_{a \beta}^{\alpha} \lambda^{b, \beta}{ }_{\gamma} \lambda^{a, \gamma}=\left(i_{\rho} \ell^{2}+\epsilon \frac{6}{\sigma_{0}}\right) \lambda_{\delta}^{b, \alpha} .
$$

The two equations above imply that

$$
\epsilon C_{\rho}+\frac{1}{2} C_{a d j}=i_{\rho} \ell^{2}+\epsilon \frac{6}{\sigma_{0}}
$$

which will turn out to be the consistency condition required by the Jacobi (super-Jacobi) identities .

\footnotetext{
${ }^{4}$ In the remainder of this section we assume that $g$ is simple.
} 
Following [13], we make the following Ansatz for the $P_{a b}^{\alpha \beta}$ tensor

$$
P_{a b}^{\alpha \beta}=\lambda_{a \gamma \gamma}^{\alpha} \lambda_{b}^{\gamma \beta}+\lambda_{b \gamma}^{\alpha} \lambda_{a}^{\gamma \beta}+y \Omega^{\alpha \beta} \eta_{a b},
$$

where $y$ is some constant to be determined. We note that the Ansatz for this tensor satisfies the symmetry conditions required by the OPE of $G^{\alpha}(z) G^{\beta}(w)$, namely, $P_{a b}^{\alpha \beta}=P_{b a}^{\alpha \beta}$ and $P_{a b}^{\alpha \beta}=-\epsilon P_{a b}^{\beta \alpha}$. Inserting (2-23) in $(2-15)$ and using (2-10), we find that

$$
y=\frac{4}{\sigma_{0}} .
$$

Substituting the expression $2-23)$ for $P_{a b}^{\alpha \beta}$ in $(2-17)$ and $\left.2-16\right)$ and using the commutation relations, we see that the condition $2-17)$ is trivially satisfied and (2-16) leads to

$$
c=\frac{3}{2} b+\gamma k \ell^{2}\left(-C_{\rho}+\frac{2}{\sigma_{0}} D\right) .
$$

Inserting $(2-23)$ in the right-hand side of $(2-18)$ and using the identities $(2-10)$ and $(2-20)$, we get

$$
\begin{aligned}
P_{c b}^{\alpha \beta} f_{d}^{a c} f_{e}^{d b}= & \frac{4}{\sigma_{0}}\left(i_{\rho} \ell^{2}-C_{a d j}\right) \Omega^{\alpha \beta} \delta_{e}^{a} \\
& +\left(\frac{2}{\sigma_{0}}+C_{\rho}+\epsilon C_{a d j}\right)\left(\lambda^{a, \beta}{ }_{\gamma} \lambda_{e}^{\gamma \alpha}-\epsilon \lambda^{a, \alpha}{ }_{\gamma} \lambda_{e}^{\gamma \beta}\right) .
\end{aligned}
$$

The consistency of this equation with $2-18$ requires that the following relations hold

$$
\begin{aligned}
\gamma & =\frac{\sigma_{0} / 2}{\left(k-i_{\rho}\right) \ell^{2}+C_{a d j}} \\
\sigma & =\frac{\sigma_{0}}{\left(k-i_{\rho}\right) \ell^{2}+C_{a d j}}\left(k \ell^{2}+\epsilon \frac{2}{\sigma_{0}}+\epsilon C_{\rho}+C_{a d j}\right) .
\end{aligned}
$$

Making repeated use of $(2-10)$ and $(2-20)$, the right-hand side of $(2-19)$ can be written as

$$
\begin{aligned}
P_{a b}^{\beta \gamma} \lambda_{\mu}^{a, \alpha} \lambda_{\delta}^{b, \mu}= & \frac{1}{\sigma_{0}}\left(\epsilon \frac{8}{\sigma_{0}}-4 \epsilon C_{\rho}-C_{a d j}\right) \\
& \left(\Omega^{\alpha \beta} \delta_{\delta}^{\gamma}-2 \Omega^{\beta \gamma} \delta_{\delta}^{\alpha}+\Omega^{\gamma \alpha} \delta_{\delta}^{\beta}\right) .
\end{aligned}
$$




\begin{tabular}{|l|l|l|l|l|l|}
\hline & $g$ & $D$ & $\check{g}$ & $\rho$ & $i_{\rho}$ \\
\hline SCA's $(\epsilon=-1)$ & $s o(n)$ & $\frac{1}{2} n(n-1)$ & $n-2$ & $n$ & 1 \\
& $s o(7)$ & 21 & 5 & $8_{s}$ & 1 \\
& $G_{2}$ & 14 & 4 & 7 & 1 \\
\hline QSCA's $(\epsilon=+1)$ & $s p(2 n)$ & $n(2 n+1)$ & $n+1$ & $2 n$ & $\frac{1}{2}$ \\
& $s l(6)$ & 35 & 6 & 20 & 3 \\
& $s o(12)$ & 66 & 10 & 32 & 4 \\
& $E_{7}$ & 133 & 18 & 56 & 6 \\
& $s p(6)$ & 21 & 4 & 14 & $\frac{5}{2}$ \\
& $s l(2)$ & 3 & 2 & 4 & 5 \\
\hline
\end{tabular}

Table 1: SCA's and QSCA's based on simple (complex) $g$ and irreducible $\rho$

Inserting this expression in $2-19)$, we can read-off another expression for $\sigma$

$$
\sigma=\sigma_{0}-\frac{\sigma_{0} / 2}{\left(k-i_{\rho}\right) \ell^{2}+C_{a d j}}\left(\epsilon \frac{8}{\sigma_{0}}-4 \epsilon C_{\rho}-C_{a d j}\right) .
$$

Equating the two expression for $\sigma$ gives us the consistency condition

$$
\epsilon C_{\rho}+\frac{1}{2} C_{a d j}=i_{\rho} \ell^{2}+\epsilon \frac{6}{\sigma_{0}} \text {. }
$$

This is the same consistency condition we found earlier in $(2-22)$.

In [13], complex forms of QSCA's and SCA's based on simple complex Lie algebras $g$ were classified. The QSCA's and SCA's for which the representation $\rho$ is irreducible are summarized in Table 1 . Using $(2-8)$ and $(2$ 9), the above consistency condition can be rewritten as

$$
\check{g}=2 i_{\rho}\left(\frac{D}{N}+\frac{3 D}{N(1+\epsilon N)}-\epsilon\right) .
$$

In this form, it is easy to verify that this condition is satisfied by all the algebras in Table 1.

Substituting the expressions for $C_{a d j}$ and $C_{\rho}$ in terms of $i_{\rho}$ and $\check{g}$, we find that the (super-)Jacobi identities determine $\gamma, \sigma, b$, and the central charge, $c$, as follows:

$$
\gamma=-\frac{N(1+\epsilon N)}{i_{\rho} D \ell^{4}\left(k-i_{\rho}-\epsilon \check{g}\right)}
$$




$$
\begin{aligned}
\sigma & =-\frac{2 N(1+\epsilon N)}{i_{\rho} D \ell^{2}\left(k-i_{\rho}-\epsilon \check{g}\right)}\left\{k+\frac{i_{\rho} D}{(1+\epsilon N)}-\epsilon \check{g}\right\} \\
b & =\frac{\epsilon k N(1+\epsilon N)}{i_{\rho} D\left(k-i_{\rho}-\epsilon \check{g}\right)}\left\{k+\frac{i_{\rho} D}{(1+\epsilon N)}-\epsilon \check{g}\right\} \\
c & =\frac{1}{2\left(k-i_{\rho}-\epsilon \check{g}\right)}\left\{3 k N(N+\epsilon) \frac{(k-\epsilon \check{g})}{D i_{\rho}}+5 k \epsilon N+2 k(D+1)\right\}(2-36)
\end{aligned}
$$

\section{Quaternionic Symmetric Spaces and Nonlinear Quasi-Superconformal Algebras}

For every simple compact Lie group $F$ (other than $S U(2)$ ), there exists a quaternionic symmetric space $W$ of the form

$$
W=\frac{F}{G \times S U(2)},
$$

which is unique (up to isomorphisms). The Lie algebra $f$ of $F$ can be given a 5-graded decomposition with respect to a suitable generator $e \in f$

$$
f=f^{-2} \oplus f^{-1} \oplus f^{0} \oplus f^{+1} \oplus f^{+2},
$$

such that $f^{-2}$ and $f^{+2}$ subspaces are one-dimensional and

$$
f^{0}=g \oplus e,
$$

where $g$ is the Lie algebra of $G$ and $e$ commutes with $g$. The generators belonging to $f^{ \pm 2}$ together with $e$ form the $s u(2)$ subalgebra of $f$. P By taking different non-compact real forms of the group $F$ and the corresponding real forms of $G$ and $S U(2)$, one obtains different non-compact quaternionic symmetric spaces $W$. As will be explained in the next section, the real forms that are relevant for the classification of QSCA's all have the non-compact $S p(2, \mathbf{R}) \cong S l(2, \mathbf{R}) \cong S U(1,1)$ factor in the isotropy group of the quotient space $W=F /(G \times S U(1,1))$. We shall denote the generators of the Lie algebra of this $S p(2, \mathbf{R})$ factor as $K_{i j}=K_{j i}(i, j, \ldots=1,2)$, the generators

\footnotetext{
${ }^{5}$ Here we should note that there exists a one-to-one correspondence between the grade +1 subspace $f^{+1}$ of $f$ and a simple Freudenthal triple system [27] associated with $f$. Furthermore, the $N=4$ SCA's with the $S U(2) \times S U(2) \times U(1)$ symmetry can be realized over quaternionic symmetric spaces of the form $W \times S U(2) \times U(1)$ 22, 29, 27, 30] or, equivalently, in terms of the corresponding Freudenthal triple systems [27]. Note also that the group manifold $S U(2) \times U(1)$ admits a quaternionic structure.
} 
of the subalgebra $g$ as $M_{a}(a, b, \ldots=1, \cdots, D=\operatorname{dim} G)$, and the coset space $F /(G \times S U(1,1))$ generators as $U_{\alpha i}(\alpha, \beta, \ldots=1, \ldots, N)$. The commutation relations of $f$ can be written in the form 32]

$$
\begin{aligned}
& {\left[U_{\alpha i}, U_{\beta j}\right]=\varepsilon_{i j} \Lambda_{\alpha \beta}^{a} M_{a}+\Omega_{\alpha \beta} K_{i j}} \\
& {\left[M_{a}, U_{\alpha i}\right]=\Lambda_{a, \alpha}{ }^{\beta} U_{\beta i}} \\
& {\left[M_{a}, M_{b}\right]=C_{a b}{ }^{c} M_{c}} \\
& {\left[K_{i j}, U_{\alpha k}\right]=\varepsilon_{i k} U_{\alpha j}+\varepsilon_{j k} U_{\alpha i}} \\
& {\left[K_{i j}, K_{k \ell}\right]=\varepsilon_{i k} K_{\ell j}+\varepsilon_{i \ell} K_{k j}+\varepsilon_{j k} K_{\ell i}+\varepsilon_{j \ell} K_{k i},}
\end{aligned}
$$

where $\Lambda_{\alpha \beta}^{a}=\Lambda_{\beta \alpha}^{a}$, and $\varepsilon_{i j}=-\varepsilon_{j i}$ and $\Omega_{\alpha \beta}=-\Omega_{\beta \alpha}$ are the symplectic invariant tensors of $s p(2)$ and $g$, respectively. Following [32], we define the tensor

$$
\Sigma_{\alpha \beta \gamma}^{\delta} \equiv \Lambda_{\alpha \beta}^{a} \Lambda_{a, \gamma} \delta .
$$

The Jacobi identities require that this tensor satisfy

$$
\Sigma_{\alpha \beta \gamma}{ }^{\delta}-\Sigma_{\gamma \alpha \beta}{ }^{\delta}=-\Omega_{\alpha \beta} \delta_{\gamma}^{\delta}-\Omega_{\gamma \alpha} \delta_{\beta}^{\delta}+2 \Omega_{\beta \gamma} \delta_{\alpha}^{\delta},
$$

and that the following conditions hold

$$
\begin{aligned}
& \Lambda_{\alpha \beta}^{a} C_{a b}^{c}+\Lambda_{\gamma \beta}^{c} \Lambda_{b, \alpha}^{\gamma}+\Lambda_{\gamma \alpha}^{c} \Lambda_{b, \beta}^{\gamma}=0 \\
& \Lambda_{c, \alpha}^{\gamma} C_{a b}^{c}+\Lambda_{a, \alpha}{ }^{\beta} \Lambda_{b, \beta}{ }^{\gamma}-\Lambda_{b, \alpha}{ }^{\beta} \Lambda_{a, \beta}^{\gamma}=0 \\
& \Lambda_{a, \alpha}^{\gamma} \Omega_{\gamma \beta}=\Lambda_{a, \beta}^{\gamma} \Omega_{\gamma \alpha} .
\end{aligned}
$$

The above equations imply that

$$
\Sigma_{\alpha \gamma \beta}^{\gamma}=(N+1) \Omega_{\alpha \beta} .
$$

The Killing metric of $f$ has the block diagonal form with the subspaces $\left\{M_{a}\right\},\left\{U_{\alpha i}\right\}$, and $\left\{K_{i j}\right\}$ being mutually orthogonal. The components $g_{a b}^{F}$ of the Killing metric for the subalgebra $g$ can be written as

$$
g_{a b}^{F}=g_{a b}+2 \Lambda_{a, \alpha}^{\beta} \Lambda_{b, \beta}{ }^{\alpha},
$$

where

$$
g_{a b}=C_{a c}{ }^{d} C_{b d}{ }^{c} .
$$


This implies that

$$
g_{a b}^{F}=\left(1+\frac{2 i_{\rho}}{\check{g}}\right) g_{a b},
$$

where $i_{\rho}$ is the Dynkin index of the representation $\rho$ of $g$ corresponding to $\left\{U_{\alpha j}\right\}$ and $\check{g}$ is the dual Coxeter number of $g$. If the Killing metric is nondegenerate, the components of the metric of a subgroup must be related to the components of the induced metric via

$$
\frac{1}{\check{f}} g_{a b}^{F}=I_{g} \frac{1}{\check{g}} g_{a b}
$$

where $I_{g}$ is the index of embedding of $G$ in $F$. The above equations are consistent if

$$
I_{g} \check{f}=\check{g}+2 i_{\rho},
$$

which holds true for all cases, as can be verified. Using the expression for $g_{a b}^{F}$ and

$$
g_{\alpha i, \beta j}^{F}=-2(N+4) \varepsilon_{i j} \Omega_{\alpha \beta},
$$

one finds that

$$
g_{a b} \Lambda_{\alpha \beta}^{b}=2(N+4) \Omega_{\beta \alpha} \Lambda_{a, \alpha}^{\gamma} .
$$

Substituting this in equation $(3-6)$ yields

$$
\begin{aligned}
& \Lambda_{\alpha \beta}^{a} \Lambda_{a, \gamma}{ }^{\sigma}-\Lambda_{\gamma \alpha}^{a} \Lambda_{a, \beta}{ }^{\sigma}= \\
& \frac{1}{2(N+4)}\left(\Omega_{\alpha \beta} \delta_{\gamma}^{\sigma}+\Omega_{\gamma \alpha} \delta_{\beta}^{\sigma}-2 \Omega_{\beta \gamma} \delta_{\alpha}^{\sigma}\right) .
\end{aligned}
$$

Modulo an overall normalization of $\Lambda_{a, \alpha}{ }^{\beta}$ (hence of the generators $M_{a}$ ), we see that this equation is identical to equation (2-10) that is a necessary and sufficient condition for the existence of nonlinear QSCA's. The required normalization of $M_{a}$ 's is simply

$$
\begin{aligned}
& M^{a} \rightarrow 2 \sqrt{\frac{N+4}{\sigma_{0}}} M^{a}=J_{0}^{a} \\
& \Lambda_{a, \alpha}^{\beta} \rightarrow 2 \sqrt{\frac{N+4}{\sigma_{0}}} \Lambda_{a, \alpha}^{\beta}=\lambda_{a, \alpha}{ }^{\beta} .
\end{aligned}
$$

We shall use this fact in the next section to give a classification of the real forms of nonlinear QSCA's. 


\section{Classification of the Real Forms of Nonlinear Quasi-Superconformal Algebras}

In the limit of infinite central charge, the nonlinear QSCA's, defined by equation (2-1), linearize, and the generators $G_{ \pm \frac{1}{2}}^{\alpha}, J_{0}^{a}, L_{ \pm 1}$, and $L_{0}$ (in the N-S moding) form a finite dimensional Lie algebra $f$. Simple nonlinear QSCA's are defined to be those for which the corresponding Lie algebra $f$ is simple. Hence, the classification of the real forms of simple nonlinear QSCA's reduces to the classification of real forms of $f$ satisfying condition (2) -10). As we showed in the previous section, this is equivalent to classifying different real forms of simple real Lie groups $F$ such that a suitable quotient is a quaternionic symmetric space. Since $L_{ \pm 1}$ and $L_{0}$ form the Lie algebra of the non-compact group $S U(1,1)$, we see that this quotient must be of the form

$$
\frac{F}{G \times S U(1,1)} .
$$

Using the well-known classification of symmetric spaces of simple Lie groups and the involution that maps grade $m(m=0, \pm 1, \pm 2)$ subspace into grade $-m$ subspace of $f$, one can determine all the real forms of simple nonlinear QSCA's. Below, we give the complete list, denoting the QSCA defined by the simple group $F$ as $Q F$ and the maximal compact subgroups of $F$ and $G$ as $K$ and $H$, respectively:

\begin{tabular}{|c|}
\hline$Q S U(m, n)$ \\
\hline$F=S U(m, n) \supset U(m-1, n-1) \times S U(1,1)$ \\
\hline$K=S(U(m) \times U(n) \times U(1))$ \\
\hline$G=U(m-1, n-1)$ \\
\hline$H=U(m-1) \times U(n-1)$ \\
\hline$N=2(m+n-2)$ \\
\hline
\end{tabular}

\begin{tabular}{|c|}
\hline$Q S L(n, \mathbf{R})$ \\
\hline$F=S L(n, \mathbf{R}) \supset G L(n-2, \mathbf{R}) \times S U(1,1)$ \\
\hline$K=S O(n)$ \\
\hline$G=G L(n-2, \mathbf{R})$ \\
\hline$H=S O(n-2)$ \\
\hline$N=2(n-2)$ \\
\hline
\end{tabular}




\begin{tabular}{|c|}
\hline$Q S O(n, m)$ \\
\hline$F=S O(n, m) \supset S O(n-2, m-2) \times S U(1,1) \times S U(1,1)$ \\
\hline$K=S O(n) \times S O(m)$ \\
\hline$G=S O(n-2, m-2) \times S U(1,1)$ \\
\hline$H=S O(n-2) \times S O(m-2) \times U(1)$ \\
\hline$N=2(n+m-4)$ \\
\hline
\end{tabular}

\begin{tabular}{|c|}
\hline$Q S O^{*}(2 n)$ \\
\hline$F=S O^{*}(2 n) \supset S O^{*}(n-4) \times S U(2) \times S U(1,1)$ \\
\hline$K=U(n)$ \\
\hline$G=S O^{*}(2 n-4) \times S U(2)$ \\
\hline$H=U(n-2) \times S U(2)$ \\
\hline$N=4(n-2)$ \\
\hline
\end{tabular}

\begin{tabular}{|c|}
\hline$Q S p(2 n, \mathbf{R})$ \\
\hline$F=S p(2 n, \mathbf{R}) \supset S p(2 n-2, \mathbf{R}) \times S p(2, \mathbf{R})$ \\
\hline$K=U(n)$ \\
\hline$G=S p(2 n-2, \mathbf{R})$ \\
\hline$H=U(n-1)$ \\
\hline$N=4(n-1)$ \\
\hline
\end{tabular}

\begin{tabular}{|c|}
\hline$Q E_{6(6)}$ \\
\hline$F=E_{6(6)} \supset S L(6, \mathbf{R}) \times S U(1,1)$ \\
\hline$K=U S p(8)$ \\
\hline$G=S L(6, \mathbf{R})$ \\
\hline$H=S O(6)$ \\
\hline$N=20$ \\
\hline
\end{tabular}

\begin{tabular}{|c|}
\hline$Q E_{6(2)}$ \\
\hline$F=E_{6(2)} \supset S U(3,3) \times S U(1,1)$ \\
\hline$K=S U(6) \times S U(2)$ \\
\hline$G=S U(3,3)$ \\
\hline$H=S U(3) \times S U(3) \times U(1)$ \\
\hline$N=20$ \\
\hline
\end{tabular}




\begin{tabular}{|c|}
\hline$Q E_{6(-14)}$ \\
\hline$F=E_{6(-14)} \supset S U(5,1) \times S U(1,1)$ \\
\hline$K=S O(10) \times U(1)$ \\
\hline$G=S U(5,1)$ \\
\hline$H=U(5)$ \\
\hline$N=20$ \\
\hline
\end{tabular}

\begin{tabular}{|c|}
\hline$Q E_{7(7)}$ \\
\hline$F=E_{7(7)} \supset S O(6,6) \times S U(1,1)$ \\
\hline$K=S U(8)$ \\
\hline$G=S O(6,6)$ \\
\hline$H=S O(6) \times S O(6)$ \\
\hline$N=32$ \\
\hline
\end{tabular}

\begin{tabular}{|c|}
\hline$Q E_{7(-5)}$ \\
\hline$F=E_{7(-5)}=S O^{*}(12) \times S U(1,1)$ \\
\hline$K=S O(12) \times S U(2)$ \\
\hline$G=S O^{*}(12)$ \\
\hline$H=U(6)$ \\
\hline$N=32$ \\
\hline
\end{tabular}

\begin{tabular}{|c|}
\hline$Q E_{7(-25)}$ \\
\hline$F=E_{7(-25)} \supset S O(10,2) \times S U(1,1)$ \\
\hline$K=E_{6} \times U(1)$ \\
\hline$G=S O(10,2)$ \\
\hline$H=S O(10) \times U(1)$ \\
\hline$N=32$ \\
\hline
\end{tabular}

\begin{tabular}{|c|}
\hline$Q E_{8(8)}$ \\
\hline$F=E_{8(8)} \supset E_{7(7)} \times S U(1,1)$ \\
\hline$K=S O(16)$ \\
\hline$G=E_{7(7)}$ \\
\hline$H=S U(8)$ \\
\hline$N=56$ \\
\hline
\end{tabular}




\begin{tabular}{|c|}
\hline$Q E_{8(-24)}$ \\
\hline$F=E_{8(-24)} \supset E_{7(-25)} \times S U(1,1)$ \\
\hline$K=E_{7} \times S U(2)$ \\
\hline$G=E_{7(-25)}$ \\
\hline$H=E_{6} \times U(1)$ \\
\hline$N=56$ \\
\hline
\end{tabular}

\begin{tabular}{|c|}
\hline$Q F_{4(4)}$ \\
\hline$F=F_{4(4)} \supset S p(6, \mathbf{R}) \times S U(1,1)$ \\
\hline$K=U S p(6) \times S U(2)$ \\
\hline$G=S p(6, \mathbf{R})$ \\
\hline$H=U(3)$ \\
\hline$N=14$ \\
\hline
\end{tabular}

\begin{tabular}{|c|}
\hline$Q G_{2(2)}$ \\
\hline$F=G_{2(2)} \supset S U(1,1) \times S U(1,1)$ \\
\hline$K=S U(2) \times S U(2)$ \\
\hline$G=S U(1,1)$ \\
\hline$H=U(1)$ \\
\hline$N=4$ \\
\hline
\end{tabular}

We see from the above list that the real forms of nonlinear QSCA's require, in general, non-compact symmetry groups $G$. The only exceptions occur for the series $S U(m, n)$ when $n=1$ (or $m=1$ ) for which $G=U(m-1)$ (or $U(n-1))$.

\section{Super-Quaternionic Symmetric Spaces and Non- linear Superconformal Algebras}

Ordinary nonlinear SCA's of the Bershadsky-Knizhnik type have generators of conformal dimension $2, \frac{3}{2}$, and 1 . Their supersymmetry generators $G_{\alpha}$ are fermionic and satisfy the OPE [13]

$$
\begin{aligned}
G^{\alpha}(z) G^{\beta}(w)= & \frac{b \Omega^{\alpha \beta}}{(z-w)^{3}}+\frac{\sigma \lambda_{a}^{\alpha \beta} J^{a}(w)}{(z-w)^{2}}+\frac{\sigma}{2} \frac{\lambda_{a}^{\alpha \beta} \partial J^{a}(w)}{(z-w)} \\
& +\frac{2 \Omega^{\alpha \beta} T(w)}{(z-w)}+\frac{\gamma P_{a b}^{\alpha \beta}: J^{a} J^{b}(w):}{(z-w)}+\cdots
\end{aligned}
$$


where $\Omega^{\alpha \beta}=\Omega^{\beta \alpha}$ and the $\lambda$ matrices are now antisymmetric

$$
\lambda_{a, \alpha \beta}=\lambda_{a, \alpha}^{\gamma} \Omega_{\gamma \beta}=-\lambda_{a, \beta \alpha}
$$

The remaining OPE's of nonlinear SCA's have the same form as those of the QSCA's. In Section 3, we established a one-to-one correspondence between simple nonlinear QSCA's and irreducible quaternionic symmetric spaces of simple Lie groups. This correspondence can be extended to a one-to-one correspondence between simple nonlinear SCA's and certain super-analogs of quaternionic symmetric spaces.

In the limit of infinite central charge, the nonlinear SCA's linearize, and the modes $G_{ \pm \frac{1}{2}}^{\alpha}, J_{0}, L_{ \pm 1}$, and $L_{0}$ (in the N-S moding) form a finite dimensional superalgebra $f$. Again, we define a nonlinear SCA to be simple if this finite dimensional superalgebra $f$ is simple. The superalgebra $f$ has a 5-graded decomposition

$$
f=f^{-2} \oplus f^{-1} \oplus f^{0} \oplus f^{+1} \oplus f^{+2}
$$

where $f^{0}=g \oplus e$ and $\left(f^{-2}, e, f^{+2}\right)$ form an $S U(1,1)$ subalgebra, as in the case of QSCA's. We denote the generators of this $S U(1,1)$ subalgebra as $K_{i j}=K_{j i}(i, j, \ldots=1,2)$, the odd generators belonging to $\left(f^{-1} \oplus f^{+1}\right)$ as $V_{\alpha i}(\alpha, \beta, \ldots=1, \cdots, N)$, and the generators of the subalgebra $g$ as $M_{a}(a, b, \ldots=1, \cdots, D)$. The super-commutation relations of $f$ can then be written in the form

$$
\begin{aligned}
& \left\{V_{\alpha i}, V_{\beta j}\right\}=\epsilon_{i j} A_{\alpha \beta}^{a} M_{a}+\Omega_{\alpha \beta} K_{i j} \\
& {\left[M_{a}, V_{\alpha i}\right]=A_{a, \alpha}{ }^{\beta} V_{\beta i}} \\
& {\left[M_{a}, M_{b}\right]=C_{a b}{ }^{c} M_{c}} \\
& {\left[K_{i j}, V_{\alpha k}\right]=\epsilon_{i k} V_{\alpha j}+\epsilon_{j k} V_{\alpha i}} \\
& {\left[K_{i j}, K_{k \ell}\right]=\epsilon_{i k} K_{\ell j}+\epsilon_{i \ell} K_{k j}+\epsilon_{j k} K_{\ell i}+\epsilon_{j \ell} K_{k i},}
\end{aligned}
$$

where $A_{\alpha \beta}^{a}=-A_{\beta \alpha}^{a}$ and $\epsilon_{i j}=-\epsilon_{j i}$, and $\Omega_{\alpha \beta}=\Omega_{\beta \alpha}$ is an invariant symmetric tensor of $g$. Defining

$$
\Sigma_{\alpha \beta \gamma}{ }^{\delta} \equiv A_{\alpha \beta}^{a} A_{a, \gamma}{ }^{\delta} .
$$

we find that the super-Jacobi conditions require the following relations

$$
\Sigma_{\alpha \beta \gamma}^{\delta}-\Sigma_{\gamma \alpha \beta}{ }^{\delta}=-\Omega_{\alpha \beta} \delta_{\gamma}^{\delta}-\Omega_{\gamma \alpha} \delta_{\beta}^{\delta}+2 \Omega_{\beta \gamma} \delta_{\alpha}^{\delta}
$$




$$
\begin{aligned}
& A_{\alpha \beta}^{a} C_{a b}{ }^{c}+A_{b, \alpha}{ }^{\gamma} A_{\gamma \beta}^{c}-A_{b, \beta}{ }^{\gamma} A_{\gamma \alpha}^{c}=0 \\
& A_{c, \alpha}{ }^{\gamma} C_{a b}{ }^{c}+A_{a, \alpha}{ }^{\beta} A_{b, \beta}^{\gamma}-A_{b, \alpha}{ }^{\beta} A_{a, \beta}{ }^{\gamma}=0 \\
& A_{a, \alpha}{ }^{\gamma} \Omega_{\gamma \beta}=-A_{a, \beta}{ }^{\gamma} \Omega_{\gamma \alpha}
\end{aligned}
$$

As in the case of QSCA's, it is easy to show that the above conditions are equivalent to the necessary and sufficient condition $(2-10)$ for the existence of nonlinear SCA's, if we identify

$$
\lambda_{a, \alpha \beta}=2 \sqrt{\frac{N+4}{\sigma_{0}}} A_{a, \alpha \beta}
$$

and

$$
J_{0}^{a}=2 \sqrt{\frac{N+4}{\sigma_{0}}} M^{a} .
$$

The supersymmetry generators $V_{\alpha i}$ are now associated with the supercoset space

$$
\frac{F}{G \times S U(1,1)}
$$

These coset spaces are the fermionic analogs of the quaternionic symmetric spaces. By taking different real forms of $F$, one gets different real forms of the super-quaternionic coset spaces, including the compact space $F_{\text {compact }} / G \times S U(2)$. However, for nonlinear SCA's, the relevant real forms are those for which the subgroup generated by $f^{+2}, f^{-2}$, and $e$ is the noncompact group $S U(1,1)$. A complete classification of the simple nonlinear SCA's, then, follows from the list of simple noncompact supergroups $F$ whose quotient with respect to their subgroup $G \times S U(1,1)$ is a superquaternionic symmetric space. In contrast to the real forms of noncompact groups where the subgroup $G$ is, in general, noncompact, one finds that the classical simple noncompact supergroups always admit a real form for which the subgroup $G$ is compact. A complete classification of the two dimensional global superconformal groups $F$ with Lie subgroups $G \times S U(1,1)$ such that $G$ is compact was given in [3]. On the other hand, a classification of the real forms of finite dimensional simple Lie superalgebras was given in [33]. Using the results of [3] and [33], one can give a complete list of real forms of classical simple superconformal groups $F$ satisfying the above conditions 


\begin{tabular}{|c|c|c|}
\hline$F$ & $G$ & $N$ \\
\hline$S L(m, \mathbf{R} / 2, \mathbf{R})$ & $S L(m, \mathbf{R}) \times \mathcal{D}$ & $2 m(m>2)$ \\
\hline$S U\left(2 m^{*} / 1,1\right)$ & $S U^{*}(2 m) \times \mathcal{D}$ & $4 m(m>1)$ \\
\hline$S U(m-p, p / 1,1)$ & $S U(m-p, p) \times U(1)$ & $2 m(m>2)$ \\
\hline$O S p(p, q / 2, \mathbf{R})$ & $S O(p, q)$ & $p+q$ \\
\hline$O S p\left(4^{*} / 2 n\right)$ & $U S p(2 n) \times S U(2)$ & $4 n$ \\
\hline$D(2,1 ; \alpha)_{1}$ & $S O(4)$ & 4 \\
$D(2,1 ; \alpha)_{2}$ & $S O(2,2)$ & 4 \\
$D(2,1 ; \alpha)_{3}$ & $S l(2, \mathbf{C})$ & 4 \\
\hline$S U(2 / 1,1)$ & $S U 2)$ & 4 \\
$S U(1,1 / 1,1)$ & $S U(1,1)$ & 4 \\
\hline$G(3)_{1}$ & $G_{2}$ & 7 \\
$G(3)_{2}$ & $G_{2(2)}$ & 7 \\
\hline$F(4)_{1}$ & $S O(7)$ & 8 \\
$F(4)_{2}$ & $S O(4,3)$ & 8 \\
\hline
\end{tabular}

Table 2: Above we give a complete list of the real forms of simple classical Lie superalgebras $F$ that define a nonlinear SCA with the symmetry group $G$. $\mathcal{D}$ denotes the one dimensional noncompact dilatation group. The maximal compact subgroup of $S U^{*}(2 m)$ is $U S p(2 m)$.

and hence a complete list of the classical simple nonlinear SCA's. In Table 2 , we give this classification, listing the superconformal groups $F$, their subgroups $G$ that occur in their Lie subgroups $G \times S U(1,1)$, and the number $N$ of the supersymmetry currents of the corresponding nonlinear SCA.

\section{Unified Realization of Quasi-Superconformal and Superconformal Algebras}

In this section, we shall give a unified realization of QSCA's (SCA's) with simple symmetry groups $G$ and irreducible representation $\rho$ in terms of KacMoody currents and free fields. We start with dimension-1 "bare" currents, $S^{a}(z)$, of the affine Lie algebra corresponding to the subgroup $G$ of $F$ and satisfying the OPE

$$
S^{a}(z) S^{b}(w)=\frac{-\frac{1}{2} k_{0} \ell^{2} \eta^{a b}}{(z-w)^{2}}+\frac{f_{c}^{a b} S^{c}(w)}{(z-w)}+\cdots,
$$


with "bare" level $k_{0}$. Next, we introduce bosons (fermions) $\psi^{\alpha}(z)$ of conformal dimension $\frac{1}{2}$, transforming in the representation $\rho$ of $G$, and satisfying the $\mathrm{OPE}$

$$
\psi^{\alpha}(z) \psi^{\beta}(w)=\frac{\Omega^{\alpha \beta}}{(z-w)}+\cdots .
$$

Finally, we introduce a scalar fields $\phi(z)$ of conformal dimension 0 (dilaton) normalized such that

$$
\partial \phi(z) \partial \phi(w)=\frac{1}{(z-w)^{2}}+\cdots
$$

We make a general Ansatz for the bosonic (fermionic) generators $G_{\alpha}(z)$ of the QSCA's (SCA's), constructed out of the above fields, of the form

$$
\begin{aligned}
G_{\alpha}(z)= & x_{1} \partial \psi_{\alpha}+x_{2} \partial \phi \psi_{\alpha}+x_{3} \lambda_{a, \alpha}{ }^{\beta} S^{a} \psi_{\beta} \\
& +x_{4} \lambda_{a, \alpha}{ }^{\beta} \lambda^{a, \gamma \delta}:: \psi_{\gamma} \psi_{\delta}: \psi_{\beta}:
\end{aligned}
$$

where $x_{1}, \ldots, x_{4}$ are some parameters to be determined. Before proceeding to calculate the OPE of two such $G$ 's, we need to derive several identities that will be useful. Using equation $(2-10)$, it is easy to show that

$$
\begin{aligned}
\lambda_{a, \alpha}{ }^{\mu} \lambda_{\beta}^{a}{ }^{\nu}: \partial \psi_{\mu} \psi_{\nu}:= & -\frac{2}{\sigma_{0}}\left(\epsilon: \partial \psi_{\alpha} \psi_{\beta}:+2: \partial \psi_{\beta} \psi_{\alpha}:+\Omega_{\alpha \beta}: \psi_{\mu} \partial \psi^{\mu}:\right) \\
& +\epsilon \frac{1}{2} \lambda_{a, \alpha \beta} \lambda^{a, \mu \nu} \partial: \psi_{\mu} \psi_{\nu}: \\
\lambda_{a, \alpha}{ }^{\mu} \lambda^{a}{ }_{\beta}{ }^{\nu}: \psi_{\mu} \psi_{\nu}:= & \epsilon\left(\frac{-6}{\sigma_{0}}: \psi_{\alpha} \psi_{\beta}:+\lambda_{a, \alpha \beta} \lambda^{a, \mu \nu}: \psi_{\mu} \psi_{\nu}:\right) .
\end{aligned}
$$

In the following calculations, we have to evaluate the product $\lambda_{a}{ }_{\beta} \lambda^{b, \beta}{ }_{\gamma} \lambda^{a, \gamma}{ }_{\delta}$. This product was encountered before in the solution of the Jacobi (superJacobi) conditions and led to the consistency condition

$$
\epsilon C_{\rho}+\frac{1}{2} C_{a d j}=i_{\rho} \ell^{2}+\epsilon \frac{6}{\sigma_{0}}
$$

Another expression that needs to be evaluated in the following calculations is $\lambda^{a}{ }_{\alpha \gamma} \lambda_{b}{ }^{\gamma \delta} \lambda^{b}{ }_{\beta}^{\mu} \lambda_{a, \mu}{ }^{\nu}: \psi_{\nu} \psi_{\delta}$ :. This expression can be evaluated in various ways. One way is to use $(2-10)$ to interchange the $\gamma$ and $\mu$ indices. Doing so leads to the expression

$$
\begin{aligned}
\lambda_{\alpha \gamma}^{a} \lambda_{b}{ }^{\gamma \delta} \lambda^{b}{ }_{\beta}^{\mu} \lambda_{a, \mu}{ }^{\nu}: \psi_{\nu} \psi_{\delta}:= & \frac{\epsilon}{4} C_{a d j} \lambda_{a, \alpha \beta} \lambda^{a, \mu \nu}: \psi_{\mu} \psi_{\nu}: \\
& +\frac{1}{\sigma_{0}}\left(-\frac{12}{\sigma_{0}}+4 C_{\rho}\right): \psi_{\alpha} \psi_{\beta}: .
\end{aligned}
$$


If we use $(2-10)$ to interchange the $\delta$ and $\mu$ indices, the expression takes the form

$$
\begin{aligned}
\lambda_{\alpha \gamma}^{a} \lambda_{b}^{\gamma \delta} \lambda^{b}{ }_{\beta}^{\mu} \lambda_{a, \mu}{ }^{\nu}: \psi_{\nu} \psi_{\delta} & :=-\left(\frac{6}{\sigma_{0}}+C_{\rho}+\frac{\epsilon}{2} C_{a d j}\right) \lambda_{a, \alpha \beta} \lambda^{a, \mu \nu}: \psi_{\mu} \psi_{\nu}: \\
& +\frac{1}{\sigma_{0}}\left(\frac{12}{\sigma_{0}}+8 C_{\rho}+3 \epsilon C_{a d j}\right): \psi_{\alpha} \psi_{\beta}: \quad(6-9)
\end{aligned}
$$

The two equations above are consistent only if

$$
-\epsilon \frac{3}{4} C_{a d j}=C_{\rho}+\frac{6}{\sigma_{0}} .
$$

(The same result can be obtained by evaluating $\lambda_{\alpha \gamma}^{a} \lambda_{b}{ }^{\gamma \delta} \lambda^{b}{ }_{\beta}^{\mu} \lambda_{a, \mu}{ }^{\nu}: \partial \psi_{\nu} \psi_{\delta}$ :, which appears in the following calculation, in a couple of different ways.) As we shall see,$(6-7)$ and $(6-10)$ are the two consistency conditions that must be satisfied for realizing QSCA's (SCA's) using our general Ansatz for the supersymmetry generators.

In analogy with the tensor $\Sigma$ defined in Sections 3 and 5 , we define a tensor $\Pi$

$$
\Pi_{\alpha \beta \gamma \delta}=\lambda_{a, \alpha \beta} \lambda_{\gamma \delta}^{a},
$$

which is symmetric (antisymmetric) in $\alpha, \beta$ and $\gamma, \delta$ for QSCA's (SCA's) and is invariant under $\alpha \beta \longleftrightarrow \gamma \delta$. A totally symmetric (antisymmetric) tensor $S$ can be constructed out of $\Pi$ through

$$
S_{\alpha \beta \gamma \delta}=\frac{1}{3}\left(\Pi_{\alpha \beta \gamma \delta}+\Pi_{\alpha \delta \beta \gamma}+\Pi_{\alpha \gamma \delta \beta}\right) .
$$

Using the identity (2-10), $S$ tensor can be written as

$$
S_{\alpha \beta \gamma \delta}=\Pi_{\alpha \beta \gamma \delta}-\frac{2}{\sigma_{0}}\left(\Omega_{\alpha \gamma} \Omega_{\beta \delta}+\epsilon \Omega_{\alpha \delta} \Omega_{\beta \gamma}\right) .
$$

We shall now derive a very important and useful identity involving the $S$ tensor. We start from two different ways of writing the commutation relations of the $\lambda$ matrices

$$
\begin{aligned}
& \lambda_{\alpha \beta}^{a} f_{a b}^{c}+\lambda_{\beta \gamma}^{c} \lambda_{b, \alpha}{ }^{\gamma}+\lambda_{\gamma \alpha}^{c} \lambda_{b, \beta}^{\gamma}=0 \\
& \lambda_{c, \alpha}{ }^{\gamma} f_{a b}{ }^{c}+\lambda_{a, \alpha}{ }^{\beta} \lambda_{b \beta}^{\gamma}-\lambda_{b, \alpha}{ }^{\beta} \lambda_{a \beta}^{\gamma}=0 .
\end{aligned}
$$


Multiplying the second equation above by $\lambda^{a}{ }_{\mu \nu} \lambda^{b}{ }_{\sigma \tau}$ and using the first equation to eliminate $\lambda^{a}{ }_{\mu \nu} f_{a b}^{c}$ gives

$$
\Pi_{\nu \rho \alpha}^{\gamma} \Pi_{\sigma \tau \mu}{ }^{\rho}+\Pi_{\rho \mu \alpha}^{\gamma} \Pi_{\sigma \tau \nu}{ }^{\rho}-\Pi_{\sigma \tau}^{\gamma} \rho \Pi_{\mu \nu \alpha}{ }^{\rho}+\Pi_{\mu \nu}^{\gamma}{ }_{\rho} \Pi_{\sigma \tau \alpha}{ }^{\rho}=0 .
$$

Rewriting the $\Pi$ 's in this identity in terms of $S$ 's, using (6-13), and symmetrizing (antisymmetrizing) in the five indices $\mu, \alpha, \sigma, \tau$, and $\nu$ gives the identity

$$
S_{\epsilon \rho(\mu \alpha} S_{\sigma \tau \nu]}^{\rho}+\frac{2}{\sigma_{0}} S_{(\mu \alpha \sigma \tau} \Omega_{\nu] \epsilon}=0
$$

where (...] means symmetrization for QSCA's and antisymmetrization for SCA's. Finally, using this identity, one finds that

$$
\begin{aligned}
& \lambda_{\alpha \gamma}^{(a} \lambda_{\beta}^{b), \gamma} \lambda_{a, \mu \nu} \lambda_{b, \rho \sigma}:: \psi^{\mu} \psi^{\nu}:: \psi^{\rho} \psi^{\sigma}::= \\
& \frac{4}{3 \sigma_{0}} \Omega_{\alpha \beta} \lambda_{a, \mu \nu} \lambda_{\rho \sigma}^{a}:: \psi^{\mu} \psi^{\nu}:: \psi^{\rho} \psi^{\sigma}::-\epsilon \frac{32}{3 \sigma_{0}} \lambda^{a, \mu \nu} \lambda_{a[\alpha}^{\rho} \psi_{\beta) \mu \nu \rho} \\
& +\frac{1}{\sigma_{0}}\left(\epsilon \frac{32}{\sigma_{0}}-16 \epsilon C_{\rho}-4 C_{a d j}\right): \partial \psi_{[\alpha} \psi_{\beta]}: \\
& +\frac{1}{\sigma_{0}}\left(\frac{-64}{\sigma_{0}}+\frac{32}{3} C_{\rho}+\epsilon 8 C_{a d j}\right) \Omega_{\alpha \beta}: \psi_{\mu} \partial \psi^{\mu}:
\end{aligned}
$$

where $[\ldots$ ) means antisymmetrization for QSCA's and symmetrization for SCA's. I $\psi_{\alpha \beta \mu \nu}$ is completely symmetric (antisymmetric) and is defined as

$$
\psi_{\alpha \beta \mu \nu} \equiv: \psi_{\alpha}: \psi_{\beta}: \psi_{\mu} \psi_{\nu}:::
$$

We are now ready to calculate the OPE of two generators $G_{\alpha}(z)$ and $G_{\beta}(w)$

$$
\begin{aligned}
& G_{\alpha}(z) G_{\beta}(w)= \\
& \frac{1}{(z-w)^{3}}\left[-2 x_{1}^{2}+x_{2}^{2}+\epsilon \frac{1}{2} k_{0} \ell^{2} C_{\rho} x_{3}^{2}-8 C_{\rho} x_{1} x_{4}\right. \\
& \left.\quad+x_{4}^{2}\left(-2 C_{\rho}^{2}-\frac{12}{\sigma_{0}} C_{\rho}+\epsilon 3 C_{a d j} C_{\rho}\right)\right] \Omega_{\alpha \beta}
\end{aligned}
$$

\footnotetext{
${ }^{6}$ For the quaternionic symmetric spaces, this identity was first written down in 32 .

${ }^{7}$ Note the difference between $[\ldots)$ and $(\ldots]$.
} 


$$
\begin{aligned}
& +\frac{1}{(z-w)^{2}}\left\{\lambda_{a, \alpha \beta} S^{a}\left(2 x_{1} x_{3}+\epsilon \frac{1}{2} C_{a d j} x_{3}^{2}+4 C_{\rho} x_{3} x_{4}\right)\right. \\
& +\lambda_{a, \alpha \beta} \lambda^{a, \mu \nu}: \psi_{\mu} \psi_{\nu}:\left[6 x_{1} x_{4}-\epsilon \frac{1}{2} k_{0} \ell^{2} x_{3}^{2}+x_{4}^{2}\left(\frac{36}{\sigma_{0}}+6 C_{\rho}\right)\right] \\
& +: \psi_{\alpha} \psi_{\beta}:\left[\frac{-24}{\sigma_{0}} x_{1} x_{4}+x_{2}^{2}+\epsilon \frac{3 k_{0} \ell^{2}}{\sigma_{0}} x_{3}^{2}\right. \\
& \left.\left.+\frac{1}{\sigma_{0}} x_{4}^{2}\left(-\frac{216}{\sigma_{0}}+12 C_{\rho}+\epsilon 18 C_{a d j}\right)\right]\right\} \\
& +\frac{1}{(z-w)}\left\{\lambda_{a, \alpha \beta} \partial S^{a}\left(x_{1} x_{3}+\epsilon \frac{1}{4} C_{a d j} x_{3}^{2}+2 C_{\rho} x_{3} x_{4}\right)\right. \\
& +\lambda_{a, \alpha \beta} \lambda^{a, \mu \nu} \partial: \psi_{\mu} \psi_{\nu}:\left[3 x_{1} x_{4}-\epsilon \frac{1}{4} k_{0} \ell^{2} x_{3}^{2}+x_{4}^{2}\left(\frac{18}{\sigma_{0}}+3 C_{\rho}\right)\right] \\
& +\partial: \psi_{\alpha} \psi_{\beta}:\left(\frac{-12}{\sigma_{0}} x_{1} x_{4}-\frac{24}{\sigma_{0}} C_{\rho} x_{4}^{2}\right) \\
& +: \partial \psi_{\alpha} \psi_{\beta}:\left[x_{2}^{2}+\epsilon \frac{k_{0} \ell^{2}}{\sigma_{0}} x_{3}^{2}+\frac{1}{\sigma_{0}} x_{4}^{2}\left(-\frac{216}{\sigma_{0}}+84 C_{\rho}+\epsilon 18 C_{a d j}\right)\right] \\
& +: \partial \psi_{\beta} \psi_{\alpha}:\left(\frac{2 k_{0} \ell^{2}}{\sigma_{0}} x_{3}^{2}-\epsilon \frac{24}{\sigma_{0}} C_{\rho} x_{4}^{2}\right) \\
& +\left(x_{1} x_{2}+2 C_{\rho} x_{2} x_{4}\right) \Omega_{\alpha \beta} \partial^{2} \phi+x_{2}^{2} \Omega_{\alpha \beta}: \partial \phi \partial \phi: \\
& +\Omega_{\alpha \beta}: \psi_{\mu} \partial \psi^{\mu}:\left[\frac{k_{0} \ell^{2}}{\sigma_{0}} x_{3}^{2}+\frac{1}{\sigma_{0}} x_{4}^{2}\left(\epsilon \frac{216}{\sigma_{0}}-\epsilon 36 C_{\rho}-18 C_{a d j}\right)\right] \\
& +\epsilon \frac{2}{\sigma_{0}} x_{3}{ }^{2} \Omega_{\alpha \beta} \lambda_{a, \mu \nu} S^{a}: \psi^{\mu} \psi^{\nu}:+\epsilon \frac{1}{2} x_{3}{ }^{2} \lambda_{\alpha \mu}^{(a}{ }_{\alpha \mu}^{b), \mu}{ }_{\beta}: S_{a} S_{b}: \\
& +\frac{\epsilon}{\sigma_{0}} x_{3}\left(-4 x_{3}+12 x_{4}\right) \lambda_{a,[\alpha}{ }^{\mu}: \psi_{\beta} \psi_{\mu}: S^{a} \\
& +\epsilon\left(-\frac{1}{2} x_{3}^{2}+3 x_{3} x_{4}\right) \lambda_{a, \mu[\alpha} \lambda_{\beta)}^{b}{ }^{\mu} \lambda_{b, \rho \sigma} S^{a}: \psi^{\rho} \psi^{\sigma}: \\
& +\frac{36}{\sigma_{0}} x_{4}{ }^{2} \lambda_{a}^{\mu \nu} \lambda^{a, \rho}{ }_{[\alpha} \psi_{\beta) \mu \nu \rho}
\end{aligned}
$$




$$
\left.+\epsilon \frac{9}{2} x_{4}{ }^{2} \lambda_{\alpha \gamma}^{(a} \lambda_{\beta}^{b), \gamma} \lambda_{a, \mu \nu} \lambda_{b, \rho \sigma}:: \psi^{\mu} \psi^{\nu}:: \psi^{\rho} \psi^{\sigma}::\right\}+\cdots(6-20)
$$

where, again, [...) means antisymmetrization for QSCA's and symmetrization for SCA's. From the $(z-w)^{-3}$ term, we read-off the constant $b$ in the algebra $2-1$ )

$$
\begin{aligned}
b= & -2 x_{1}{ }^{2}+x_{2}{ }^{2}+\epsilon \frac{1}{2} k_{0} \ell^{2} C_{\rho} x_{3}{ }^{2}-8 C_{\rho} x_{1} x_{4} \\
& +x_{4}{ }^{2} C_{\rho}\left(-\frac{12}{\sigma_{0}}-2 C_{\rho}+3 \epsilon C_{a d j}\right) .
\end{aligned}
$$

By looking at the $(z-w)^{-2}$ terms in equations $(2-1)$ and $(6-20)$, we see that the "full" current, $J^{a}(z)$, has two terms, $S^{a}(z)$ and $\lambda_{\mu \nu}^{a}: \psi^{\mu} \psi^{\nu}:(z)$. In fact, the normalized $J^{a}(z)$ is given by

$$
J^{a}(z)=S^{a}(z)+\frac{1}{2} \lambda^{a}{ }_{\mu \nu}: \psi^{\mu} \psi^{\nu}:(z),
$$

satisfying the required $\mathrm{OPE}$

$$
J^{a}(z) J^{b}(w)=\frac{-\frac{1}{2} k \ell^{2} \eta^{a b}}{(z-w)^{2}}+\frac{f_{c}^{a b} J^{c}(w)}{(z-w)}+\cdots,
$$

where the "full" level $k$ is given by $k=k_{0}+i_{\rho}$. Knowing the form of $J^{a}(z)$, we find

$$
\begin{aligned}
\sigma & =2 x_{1} x_{3}+\epsilon \frac{1}{2} C_{a d j} x_{3}^{2}+4 C_{\rho} x_{3} x_{4} \\
& =12 x_{1} x_{4}-\epsilon k_{0} \ell^{2} x_{3}^{2}+2 x_{4}^{2}\left(\frac{36}{\sigma_{0}}+6 C_{\rho}\right) .
\end{aligned}
$$

The requirement that the $: \psi_{\alpha} \psi_{\beta}$ : term in $(6-20)$ be absent leads to

$$
\begin{aligned}
-\frac{24}{\sigma_{0}} x_{1} x_{4}+ & x_{2}^{2}+\epsilon \frac{3 k_{0} \ell^{2}}{\sigma_{0}} x_{3}^{2} \\
& +\frac{1}{\sigma_{0}} x_{4}^{2}\left(-\frac{216}{\sigma_{0}}+12 C_{\rho}+\epsilon 18 C_{a d j}\right)=0 .
\end{aligned}
$$

The term bilinear in the currents, then, takes the form

$$
\begin{aligned}
P_{\alpha \beta}^{a b}: J_{a} J_{b}:= & \lambda_{\alpha \rho}^{(a} \lambda^{b), \rho}{ }_{\beta}: S_{a} S_{b}: \\
& +\frac{1}{4} \lambda^{(a}{ }_{\alpha \gamma} \lambda^{b), \gamma}{ }_{\beta} \lambda_{a, \mu \nu} \lambda_{b, \rho \sigma}:: \psi^{\mu} \psi^{\nu}:: \psi^{\rho} \psi^{\sigma}:: \\
& +\frac{4}{\sigma_{0}} \Omega_{\alpha \beta}: J^{a} J_{a}: \\
& +\lambda^{(a}{ }_{\alpha \rho} \lambda^{b), \rho}{ }_{\beta} \lambda_{b, \mu \nu} S_{a}: \psi^{\mu} \psi^{\nu}:
\end{aligned}
$$


We now focus on the $(z-w)^{-1}$ terms. Noting that the term $\lambda_{a,[\alpha}{ }^{\mu}: \psi_{\beta)} \psi_{\mu}$ : $S^{a}$ has to be absent, we find the condition

$$
x_{4}=\frac{1}{3} x_{3} .
$$

Taking into account $6-25), 6-27)$, and $(6-28)$, the $(z-w)^{-1}$ terms of the product $G_{\alpha}(z) G_{\beta}(w)$ can be written as

$$
\begin{aligned}
& \frac{1}{2} \sigma \lambda_{a, \alpha \beta} \partial J^{a}+\gamma P_{\alpha \beta}^{a b}: J_{a} J_{b}:+\frac{4}{\sigma_{0}} x_{3}^{2} \lambda_{a}^{\mu \nu} \lambda_{[\alpha}^{a, \rho} \psi_{\beta) \mu \nu \rho} \\
& +\epsilon \frac{3}{8} x_{3}{ }^{2} \lambda^{(a}{ }_{\alpha \gamma} \lambda^{b), \gamma}{ }_{\beta} \lambda_{a, \mu \nu} \lambda_{b, \rho \sigma}:: \psi^{\mu} \psi^{\nu}:: \psi^{\rho} \psi^{\sigma}:: \\
& +\frac{1}{\sigma_{0}}\left(-4 x_{1} x_{3}-\frac{8}{3} C_{\rho} x_{3}^{2}\right) \partial: \psi_{\alpha} \psi_{\beta}: \\
& +\left[x_{2}^{2}+\frac{1}{\sigma_{0}} x_{3}^{2}\left(\epsilon k_{0} \ell^{2}-\frac{24}{\sigma_{0}}+\frac{28}{3} C_{\rho}+\epsilon 2 C_{a d j}\right)\right]: \partial \psi_{\alpha} \psi_{\beta}: \\
& +\frac{1}{\sigma_{0}} x_{3}^{2}\left(2 k_{0} \ell^{2}-\epsilon \frac{8}{3} C_{\rho}\right): \partial \psi_{\beta} \psi_{\alpha}: \\
& +\frac{1}{\sigma_{0}} x_{3}{ }^{2}\left(k_{0} \ell^{2}+\epsilon \frac{24}{\sigma_{0}}-\epsilon 4 C_{\rho}-2 C_{a d j}\right) \Omega_{\alpha \beta}: \psi_{\mu} \partial \psi^{\mu}: \\
& +\left(x_{1} x_{2}+\frac{2}{3} C_{\rho} x_{2} x_{3}\right) \Omega_{\alpha \beta} \partial^{2} \phi+x_{2}^{2} \Omega_{\alpha \beta}: \partial \phi \partial \phi: \\
& -\epsilon \frac{2}{\sigma_{0}} x_{3}^{2} \Omega_{\alpha \beta}: S^{a} S_{a}:
\end{aligned}
$$

Comparing the coefficients of the nonlinear terms in the above equation and in $(6-20)$, we find

$$
\gamma=\epsilon \frac{1}{2} x_{3}^{2}
$$

Making use of the identity $(6-18)$ in equation $(6-29)$ and requiring that the terms : $\partial \psi_{\alpha} \psi_{\beta}$ : and : $\partial \psi_{\beta} \psi_{\alpha}$ : be absent in the resulting equation leads to two constraints

$$
\frac{-4}{\sigma_{0}} x_{1} x_{3}+x_{2}^{2}+x_{3}^{2} \frac{1}{\sigma_{0}}\left(\epsilon k_{0} \ell^{2}-\frac{12}{\sigma_{0}}+\frac{2}{3} C_{\rho}+\epsilon \frac{1}{2} C_{a d j}\right)=0
$$




$$
-4 \epsilon x_{1} x_{3}+x_{3}^{2}\left(2 k_{0} \ell^{2}-\epsilon \frac{12}{\sigma_{0}}+\epsilon \frac{2}{3} C_{\rho}+\frac{3}{2} C_{a d j}\right)=0 .
$$

We can read-off the energy-momentum tensor to be

$$
T(z)=y_{1}: \partial \phi \partial \phi:+y_{2} \partial^{2} \phi+y_{3}: \psi_{\mu} \partial \psi^{\mu}:+y_{4}: S^{a} S_{a}:
$$

where

$$
\begin{aligned}
y_{1} & =\frac{1}{2} x_{2}^{2} \\
y_{2} & =\frac{1}{2} x_{2}\left(x_{1}+\frac{2}{3} x_{3} C_{\rho}\right) \\
y_{3} & =\frac{1}{2 \sigma_{0}} x_{3}^{2}\left(k_{0} \ell^{2}+C_{a d j}\right) \\
y_{4} & =-\frac{\epsilon}{\sigma_{0}} x_{3}^{2} .
\end{aligned}
$$

For the $J^{a}(z) G^{\alpha}(w)$ to agree with $(2-1)$, we must have

$$
-x_{1}+\epsilon x_{3}\left(\frac{1}{2} k_{0} \ell^{2}+\frac{1}{3} i_{\rho} \ell^{2}+\frac{1}{3} C_{a d j}\right)=0 .
$$

The requirement that $J^{a}(z)$ and $G^{\alpha}(z)$ be primary fields of dimension 1 and $\frac{3}{2}$, respectively, leads to the conditions

$$
\begin{aligned}
& y_{1}=\frac{1}{2} \\
& y_{3}=\epsilon \frac{1}{2} \\
& y_{4}=\frac{-1}{k_{0} \ell^{2}+C_{a d j}} \\
& -2 x_{2} y_{2}+\epsilon y_{3}\left(2 x_{1}+\frac{4}{3} x_{3} C_{\rho}\right)=0 .
\end{aligned}
$$

Finally, the central charge, $c$, of the theory is given as

$$
c=1-12 y_{2}^{2}-\epsilon \frac{1}{2} N+\frac{k_{0} \ell^{2} D}{k_{0} \ell^{2}+C_{a d j}} .
$$

Solving the above equations yields the following expressions for $x_{1}, \ldots, x_{4}$

$$
\begin{aligned}
x_{1} & =x_{3}\left(\epsilon \frac{1}{2} k_{0} \ell^{2}-\frac{6}{\sigma_{0}}-\frac{1}{3} C_{\rho}\right) \\
x_{2}^{2} & =1 \\
x_{3}^{2} & =\frac{\epsilon \sigma_{0}}{k_{0} \ell^{2}+C_{a d j}} \\
x_{4} & =\frac{1}{3} x_{3}
\end{aligned}
$$


and the following for $y_{1}, \ldots, y_{4}$

$$
\begin{aligned}
y_{1} & =\frac{1}{2} \\
y_{2} & =\frac{1}{2} x_{2} x_{3}\left(\epsilon \frac{1}{2} k_{0} \ell^{2}-\frac{6}{\sigma_{0}}+\frac{1}{3} C_{\rho}\right) \\
y_{3} & =\epsilon \frac{1}{2} \\
y_{4} & =\frac{-1}{k_{0} \ell^{2}+C_{a d j}} .
\end{aligned}
$$

Thus, we find the following expressions for the constants $\gamma$ and $\sigma$ of the algebra

$$
\begin{aligned}
& \gamma=\frac{\sigma_{0} / 2}{k_{0} \ell^{2}+C_{a d j}} \\
& \sigma=\frac{\sigma_{0}}{k_{0} \ell^{2}+C_{a d j}}\left(k_{0} \ell^{2}-\epsilon \frac{16}{\sigma_{0}}\right),
\end{aligned}
$$

along with the two consistency conditions

$$
\epsilon C_{\rho}+\frac{1}{2} C_{a d j}=i_{\rho} \ell^{2}+\epsilon \frac{6}{\sigma_{0}}
$$

and

$$
\epsilon \frac{3}{4} C_{a d j}=-C_{\rho}-\frac{6}{\sigma_{0}} .
$$

These consistency conditions are the same as the ones we had predicted earlier in $(6-7)$ and $(6-10)$. Using these consistency conditions, we can show that the expressions $(6-46),(6-47),(6-21)$, and $(6-43)$ for $\gamma, \sigma, b$, and $c$ are the same as those found in Section 2, namely, (2-27), (2-28) (or $(2-30)$ ), (2-14), and (2-25), respectively. As mentioned before the first consistency condition, (6-48), is satisfied by all the QSCA's $(\epsilon=+1)$ and all the SCA's $(\epsilon=-1)$ with simple $G$ and irreducible $\rho$. Using $C_{\rho}=\frac{i_{\rho} \ell^{2} D}{N}$ and $C_{a d j}=-\epsilon \ell^{2} \check{g}$, the second consistency condition, 6-49), can be written as

$$
\check{g}=\frac{4 i_{\rho} D}{3 N}\left(\frac{\epsilon N-2}{\epsilon N+1}\right) .
$$

In this form, it is easy to verify that $(6-50)$ is satisfied by all the QSCA's $(\epsilon=+1)$ and all the SCA's $(\epsilon=-1)$ with simple symmetry groups $G$ 
and irreducible $\rho$ except for QSCA's with $S p(2 n, R)$ symmetry and SCA's with $S O(n-m, m)$ symmetry. Hence, the general solution we obtained to the constraints starting from the general Ansatz (6-4) must not be applicable in these cases. The reason for this is that, in the representation $\rho$, the completely symmetric (antisymmetric) invariant tensor $S_{\alpha \beta \gamma \delta}$ vanishes for $S p(2 n)(S O(n))$ groups. Thus, for these algebras, the normal ordered trilinear term in the dimension- $\frac{1}{2}$ fields is absent in the general Ansatz, (64). For these two families of algebras, $(2-10)$ admits a very special solution (that is not true for the other algebras), which is

$$
\lambda^{a, \alpha \beta} \lambda_{a \delta}^{\gamma}=\frac{-2}{\sigma_{0}}\left(\epsilon \Omega^{\alpha \gamma} \delta_{\delta}^{\beta}+\Omega^{\beta \gamma} \delta_{\delta}^{\alpha}\right)
$$

Using this, we see that the $3-\psi$ term in $(6-4)$ can be written as

$$
\lambda^{a}{ }_{\alpha}^{\beta} \lambda_{a, \gamma \delta}:: \psi^{\gamma} \psi^{\delta}: \psi_{\beta}:=2 C_{\rho} \partial \psi_{\alpha} .
$$

So, the most general Ansatz for the generators $G_{\alpha}(z)$ for the $S p(2 n)(S O(n))$ algebras takes the form

$$
G_{\alpha}(z)=\hat{x}_{1} \partial \psi_{\alpha}+\hat{x}_{2} \partial \phi \psi_{\alpha}+\hat{x}_{3} \lambda_{a, \alpha}^{\beta} S^{a} \psi_{\beta}
$$

where $\hat{x}_{1}, \ldots, \hat{x}_{3}$ are some parameters to be determined. In calculating the OPE of two such generators, we need to evaluate the product $f_{a b c} \lambda_{\alpha \gamma}^{a} \lambda^{b, \gamma}{ }_{\beta}$, which can be done in two different ways. One way is to antisymmetrize in the $a$ and $b$ indices and then use the commutation relations. Doing this leads to the answer $\frac{1}{2} C_{a d j} \lambda_{c, \alpha \beta}$. Another way is to use the commutation relations to rewrite $f_{a b c} \lambda^{b, \gamma}{ }_{\beta}$ (or $f_{a b c} \lambda^{a}{ }_{\alpha \gamma}$ ) in terms of an antisymmetric product of two $\lambda$-matrices and then use 6 - 51). This gives the answer $\frac{2}{\sigma_{0}}(\epsilon 2+N) \lambda_{c, \alpha \beta}$. Thus, we find the consistency condition

$$
C_{a d j}=\frac{4}{\sigma_{0}}(\epsilon 2+N) .
$$

The identity (6-51) also implies that

$$
\lambda_{a, \alpha \beta} \lambda^{a, \mu \nu}: \psi_{\mu} \psi_{\nu}:=\frac{4}{\sigma_{0}}: \psi_{\alpha} \psi_{\beta}: \text {. }
$$

Using the two equations above, the product of $G_{\alpha}(z)$ with $G_{\beta}(w)$ becomes

$$
\begin{aligned}
& G_{\alpha}(z) G_{\beta}(w)= \\
& \frac{1}{(z-w)^{3}}\left(-2 \hat{x}_{1}^{2}+\hat{x}_{2}^{2}+\epsilon \frac{1}{2} k_{0} \ell^{2} C_{\rho} \hat{x}_{3}^{2}\right) \Omega_{\alpha \beta}
\end{aligned}
$$




$$
\begin{aligned}
+\frac{1}{(z-w)^{2}}[ & \lambda_{a, \alpha \beta} S^{a}\left(2 \hat{x}_{1} \hat{x}_{3}+\epsilon \frac{1}{2} C_{a d j} \hat{x}_{3}^{2}\right) \\
& \left.+\lambda_{a, \alpha \beta} \lambda^{a, \mu \nu}: \psi_{\mu} \psi_{\nu}:\left(\frac{\sigma_{0}}{4} \hat{x}_{2}^{2}+\epsilon \frac{1}{4} k_{0} \ell^{2} \hat{x}_{3}^{2}\right)\right] \\
+\frac{1}{(z-w)}[ & \lambda_{a, \alpha \beta} \partial S^{a}\left(\hat{x}_{1} \hat{x}_{3}+\epsilon \frac{1}{4} C_{a d j} \hat{x}_{3}^{2}\right) \\
& -\epsilon \frac{1}{4} k_{0} \ell^{2} \hat{x}_{3}{ }^{2} \lambda_{a, \alpha \beta} \lambda^{a, \mu \nu} \partial: \psi_{\mu} \psi_{\nu}: \\
& +: \partial \psi_{\alpha} \psi_{\beta}:\left(\hat{x}_{2}{ }^{2}+\epsilon \frac{k_{0} \ell^{2}}{\sigma_{0}} \hat{x}_{3}{ }^{2}\right) \\
& +\frac{2 k_{0} \ell^{2}}{\sigma_{0}} \hat{x}_{3}{ }^{2}: \partial \psi_{\beta} \psi_{\alpha}:+\frac{k_{0} \ell^{2}}{\sigma_{0}} \hat{x}_{3}{ }^{2} \Omega_{\alpha \beta}: \psi_{\mu} \partial \psi^{\mu}: \\
& +\hat{x}_{1} \hat{x}_{2} \Omega_{\alpha \beta} \partial^{2} \phi+\hat{x}_{2}{ }^{2} \Omega_{\alpha \beta}: \partial \phi \partial \phi: \\
& +\epsilon \frac{2}{\sigma_{0}} \hat{x}_{3}{ }^{2} \Omega_{\alpha \beta} \lambda_{a, \mu \nu}: \psi^{\mu} \psi^{\nu}: S^{a} \\
& +\epsilon \frac{1}{2} \hat{x}_{3}{ }^{2} \lambda^{(a}{ }_{\alpha \mu} \lambda^{b), \mu}{ }_{\beta}: S_{a} S_{b}: \\
& \left.-\epsilon \frac{2}{\sigma_{0}} \hat{x}_{3}{ }^{2} \lambda_{a,[\alpha}{ }^{\mu}: \psi_{\beta}{ }_{\mu} \psi_{\mu}: S^{a}\right]+\cdots
\end{aligned}
$$

where, again, [...) means antisymmetrization for QSCA's and symmetrization for SCA's. Looking at the $(z-w)^{-3}$ term, we read-off the constant $b$ in the algebra $(2-1)$

$$
b=-2 \hat{x}_{1}^{2}+\hat{x}_{2}^{2}+\epsilon \frac{1}{2} k_{0} \ell^{2} C_{\rho} \hat{x}_{3}^{2} .
$$

The "full" current, $J^{a}(z)$, is still given by the formula (6-22), satisfying the same OPE, with same "full" level $k=k_{0}+i_{\rho}$. Knowing the form of $J^{a}(z)$, we find

$$
\begin{aligned}
\sigma & =2 \hat{x}_{1} \hat{x}_{3}+\epsilon \frac{1}{2} C_{a d j} \hat{x}_{3}{ }^{2} \\
& =\frac{\sigma_{0}}{2} \hat{x}_{2}{ }^{2}+\epsilon \frac{1}{2} k_{0} \ell^{2} \hat{x}_{3}{ }^{2} .
\end{aligned}
$$


Since the "full" current has the same form as in (6-22), the formula for the nonlinear term $P_{\alpha \beta}^{a b}: J_{a} J_{b}$ : still has the same form as in the general case, given by $(6-27)$. However, in the present case, with the help of (6-51), it can be simplified further to

$$
\begin{aligned}
P_{\alpha \beta}^{a b}: J_{a} J_{b}:= & \lambda_{\alpha \rho}^{(a} \lambda_{\beta}^{b), \rho}: S_{a} S_{b}:+\frac{4}{\sigma_{0}} \Omega_{\alpha \beta}: S^{a} S_{a}: \\
& -\frac{4}{\sigma_{0}} \lambda_{a,[\alpha}{ }^{\mu}: \psi_{\beta} \psi_{\mu}: S^{a} \\
& +\frac{4}{\sigma_{0}} \Omega_{\alpha \beta} \lambda_{a, \mu \nu} S^{a}: \psi^{\mu} \psi^{\nu}: \\
& +\frac{4}{\sigma_{0}{ }^{2}}(\epsilon 2+N): \partial \psi_{[\alpha} \psi_{\beta)}: \\
& -\frac{1}{\sigma_{0}{ }^{2}}(16+\epsilon 8 N) \Omega_{\alpha \beta}: \psi_{\mu} \partial \psi^{\mu}: .
\end{aligned}
$$

Using this, we can write the single pole terms in $(6-56)$ as

$$
\begin{aligned}
& \frac{1}{2} \sigma \lambda_{\alpha \beta}^{a} \partial J_{a}+\gamma P_{\alpha \beta}^{a b}: J_{a} J_{b}: \\
& +\left[\frac{1}{2} \hat{x}_{2}^{2}-\hat{x}_{3}^{2} \frac{1}{\sigma_{0}}\left(\frac{2}{\sigma_{0}}(2+\epsilon N)+\epsilon \frac{1}{2} k_{0} \ell^{2}\right)\right]: \partial \psi_{[\alpha} \psi_{\beta)}: \\
& +\hat{x}_{1} \hat{x}_{2} \Omega_{\alpha \beta} \partial^{2} \phi+\hat{x}_{2}^{2} \Omega_{\alpha \beta}: \partial \phi \partial \phi:-\epsilon \frac{2}{\sigma_{0}} \hat{x}_{3}^{2} \Omega_{\alpha \beta}: S^{a} S_{a}: \\
& +\hat{x}_{3}{ }^{2} \frac{1}{\sigma_{0}}\left[\frac{4}{\sigma_{0}}(\epsilon 2+N)+k_{0} \ell^{2}\right] \Omega_{\alpha \beta}: \psi_{\mu} \partial \psi^{\mu}:
\end{aligned}
$$

¿From the single pole term of $(6-56)$ and $(6-61)$, we conclude

$$
\gamma=\epsilon \frac{1}{2} \hat{x}_{3}^{2}
$$

The absence of the term : $\partial \psi_{[\alpha} \psi_{\beta]}:$ requires that

$$
\frac{1}{2} \hat{x}_{2}^{2}-\hat{x}_{3}^{2} \frac{1}{\sigma_{0}}\left(\frac{2}{\sigma_{0}}(2+\epsilon N)+\epsilon \frac{1}{2} k_{0} \ell^{2}\right)=0 .
$$

Finally, $T(z)$ still has the form

$$
T(z)=\hat{y}_{1}: \partial \phi \partial \phi:+\hat{y}_{2} \partial^{2} \phi+\hat{y}_{3}: \psi_{\mu} \partial \psi^{\mu}:+\hat{y}_{4}: S^{a} S_{a}:
$$


where now

$$
\begin{aligned}
\hat{y}_{1} & =\frac{1}{2} \hat{x}_{2}^{2} \\
\hat{y}_{2} & =\frac{1}{2} \hat{x}_{1} \hat{x}_{2} \\
\hat{y}_{3} & =\hat{x}_{3}{ }^{2} \frac{1}{\sigma_{0}}\left[\frac{2}{\sigma_{0}}(\epsilon 2+N)+\frac{1}{2} k_{0} \ell^{2}\right] \\
\hat{y}_{4} & =-\frac{\epsilon}{\sigma_{0}} \hat{x}_{3}^{2} .
\end{aligned}
$$

For the $J^{a}(z) G^{\alpha}(w)$ to agree with $(2-1)$, we must have

$$
\hat{x}_{1}=\epsilon \frac{1}{2} k_{0} \ell^{2} \hat{x}_{3} \text {. }
$$

The requirement that $J^{a}(z)$ and $G^{\alpha}(z)$ be primary fields of dimension 1 and $\frac{3}{2}$, respectively, implies further

$$
\begin{aligned}
& \hat{y}_{1}=\frac{1}{2} \\
& \hat{y}_{3}=\epsilon \frac{1}{2} \\
& \hat{y}_{4}=\frac{-1}{k_{0} \ell^{2}+C_{a d j}} \\
& -2 \hat{x}_{2} \hat{y}_{2}+\epsilon 2 \hat{x}_{1} \hat{y}_{3}=0 .
\end{aligned}
$$

Finally, the central charge, $c$, is still given as

$$
c=1-12 \hat{y}_{2}^{2}-\epsilon \frac{1}{2} N+\frac{k_{0} \ell^{2} D}{k_{0} \ell^{2}+C_{a d j}} .
$$

Solving the above equations gives the following expressions for $\hat{x}_{1}, \ldots, \hat{x}_{3}$

$$
\begin{aligned}
\hat{x}_{1} & =\epsilon \frac{1}{2} k_{0} \ell^{2} \hat{x}_{3} \\
\hat{x}_{2}^{2} & =1 \\
\hat{x}_{3}^{2} & =\frac{\epsilon \sigma_{0}}{k_{0} \ell^{2}+C_{a d j}}
\end{aligned}
$$

and the following for $\hat{y}_{1}, \ldots, \hat{y}_{4}$

$$
\hat{y}_{1}=\frac{1}{2}
$$




$$
\begin{aligned}
& \hat{y}_{2}=\epsilon \frac{1}{4} k_{0} \ell^{2} \hat{x}_{2} \hat{x}_{3} \\
& \hat{y}_{3}=\epsilon \frac{1}{2} \\
& \hat{y}_{4}=\frac{-1}{k_{0} \ell^{2}+C_{a d j}} .
\end{aligned}
$$

Hence, we find the following expressions for the constants $\gamma$ and $\sigma$ of the algebra

$$
\begin{aligned}
\gamma & =\frac{\sigma_{0} / 2}{k_{0} \ell^{2}+C_{a d j}} \\
\sigma & =\frac{\sigma_{0}}{k_{0} \ell^{2}+C_{a d j}}\left(k_{0} \ell^{2}+\frac{1}{2} C_{a d j}\right),
\end{aligned}
$$

along with the consistency condition

$$
C_{a d j}=\frac{4}{\sigma_{0}}(\epsilon 2+N),
$$

which had been predicted earlier in $(6-54)$. Using $(2-8)$ and $(2-9)$, this condition can be written as

$$
\check{g}=\frac{2 i_{\rho} D}{N}\left(\frac{2+\epsilon N}{1+\epsilon N}\right) .
$$

It is easy to verify that this condition is satisfied by the QSCA's with $S p(2 n, R)$ symmetry $(\epsilon=+1)$ and SCA's with $S O(n-m, m)$ symmetry $(\epsilon=-1)$. Using the consistency conditions $(2-31)$ and $(6-79)$, it is easy to verify that the expressions $6-77),(6-78),(6-57)$, and $(6-74)$ for $\gamma$, $\sigma, b$, and $c$ are the same as $(2-27),(2-28)$ (or $(2-30),(2-14)$, and $(2-$ 25), respectively.

\section{Discussion and Conclusions}

Above, we have given a complete classification of the real forms of simple nonlinear QSCA's and simple classical nonlinear SCA's. We also presented a unified realization of these algebras with simple symmetry group $G$ and irreducible representation $\rho$. The ingredients of this realization are some "bare" affine currents, a dilaton and free bosons or fermions corresponding to quaternionic or super-quaternionic symmetric spaces of Lie groups or supergroups, respectively. Our method yields realizations for all allowed 
values of central charges of these algebras as functions of the bare levels of affine currents. The realizations of QSCA's and SCA's with non-simple symmetry groups as well as those of SQSCA's will be given elsewhere [31].

The exceptional nonlinear $N=8$ and $N=7$ SCA's with $\operatorname{Spin}(7)$ and $G_{2}$ symmetry were studied in [18] using coset space methods. There, it was shown that there exist unique consistent realizations of these algebras over the coset spaces $S O(8) \times U(1) / S O(7)$ and $S O(7) \times U(1) / G_{2}$ with central charges $c=84 / 11$ and $c=5$, respectively. This is to be contrasted with the realizations given above that lead to all allowed values of the central charges. 8 As argued in [18], the exceptional SCA's may arise as hidden symmetries in certain compactifications of superstring theories or of M-theory. They may also be relevant for a stringy description of the octonionic soliton solutions of heterotic string theory [34, 35, 36] and may underlie some exceptional superstring theories.

On the other hand, the nonlinear QSCA's can be interpreted as symmetry groups of two dimensional extended supergravity theories with or without matter couplings [37]. These nonlinear symmetry algebras combine an "internal" Virasoro symmetry with the affine extensions of the duality symmetry groups of corresponding four dimensional supergravity theories. The transition from the affine symmetry groups of two dimensional supergravity theories to the nonlinear QSCA's with an internal Virasoro algebra is achieved via Polyakov's soldering procedure [21] that replaces their affine $S l(2, \mathbf{R})$ symmetry with the Virasoro symmetry [37]. This is also expected from the results of Romans 223] who argued that the nonlinear QSCA's should be obtainable from affine Lie algebras via Drinfeld-Sokolov hamiltonian reduction. Further support for this interpretation comes from the recent results of Julia and Nicolai [38] who showed that the 2-d gravity coupled to a nonlinear sigma model and a dilaton has the semidirect product of the Witt algebra with an affine Lie algebra as its symmetry algebra. (Witt algebra is the centerless Virasoro algebra.)

In [39] it was shown that the light-cone actions of superstring theories in the Green-Schwarz formalism have $N=8$ supersymmetry. Coupled with the fact that these actions are also conformally invariant, this implies that thay must be invariant under some $N=8$ superconformal symmetry algebra. Later the Green-Schwarz superstring actions were shown to

\footnotetext{
${ }^{8}$ The other realization of these algebras in terms of a single boson and free fermions given in [18] corresponds to the degenerate case of taking the "bare" levels $k_{0}$ of the dimension one currents to be zero in our construction.
} 
have the $N=8$ soft algebra of reference [10] as a constraint algebra [11]. More recently, Berkovits gave a covariant formulation of the Green-Schwarz superstring that has manifest space-time (target-space) supersymmetry as well as $N=2$ world-sheet supersymmetry 40]. This formulation is especially well-suited for studying four dimensional compactifications that make super-Poincare invariance manifest. Using Berkovits' formulation De Boer and Skenderis calculated the low-energy action of the heterotic superstring in $N=1$ superspace, which is simply the old minimal supergravity coupled to the tensor multiplet [41. These results suggest that the nonlinear superconformal algebras may play an important role in the understanding of the dynamics of superstrings in the Green-Schwarz and Berkovits formulation.

\section{References}

[1] M. B. Green, J. H. Schwarz and E. Witten, Superstring Theory, Cambridge: Cambridge Univ. Press, 1987.

[2] V. Kac, Func. Anal. Appl. 9 (1975 ) 91.

[3] M. Günaydin, G. Sierra and P. K. Townsend, Nucl. Phys. B274 (1986) 429.

[4] P. Ramond and J. H. Schwarz, Phys. Lett. 64B (1976) 75.

[5] L. Alvarez-Gaumé and D. Z. Freedman, Phys. Lett. 94B (1980) 171; Phys. Rev. D22 (1980) 846; Commun. Math. Phys. 80 (1981) 443.

[6] P. Spindel, A. Sevrin, W. Troost and A. van Proeyen, Nucl. Phys. B308 (1988) 662, ibid. 311 (1988) 465.

[7] Z. Hasciewicz, K. Thielemans and W. Troost, J. Math. Phys. 31 (1990) 744 .

[8] V. G. Knizhnik, Theor. Math. Phys. 66 (1986) 68.

[9] M. Bershadsky, Phys. Lett. 174B (1986) 285.

[10] F. Englert, A. Sevrin, W. Troost, A. van Proeyen and P. Spindel, J.Math.Phys.29(1988) 281.

[11] N. Berkovits, Nucl. Phys. B358 (1991) 169. 
[12] L. Brink, M. Cederwall and C. Preitschopf, Phys. Lett. 311B (1993) 76; M. Cederwall and C. R. Preitschopf, Commun. Math. Phys. 167 (1995) 373; J. A. H. Samtleben, Nucl. Phys. B453 (1995) 429.

[13] E. S. Fradkin and V. Yu. Linetsky, Phys. Lett. 275B (1992) 345; ibid. 282 (1992) 352.

[14] P. Bowcock, Nucl. Phys. B381 (1992) 415.

[15] K. Ito, J. O. Madsen and J. L. Petersen, Phys. Lett. 318B (1993) 315; Nucl. Phys. B398 (1993) 425.

[16] P. Mathieu, Phys. Lett. 218B (1989) 185.

[17] K. Schoutens, Nucl. Phys. B292 (1987) 150; ibid. 295 (1988) 634; ibid. 314 (1989) 519.

[18] M. Günaydin and S. V. Ketov, Nucl. Phys. B467 (1996) 215.

[19] See P. Bouwknegt and K. Schoutens, "W-Symmetry and Conformal Field Theory", Physics Reports 223 (1993) 183 and the references therein.

[20] A. B. Zamolodchikov, Theor. Mat. Fiz. 65 (1985) 347.

[21] A. Polyakov, Int. J. Mod. Phys. A5 (1990) 833.

[22] M. Bershadsky, Commun. Math. Phys. 139 (1993) 67.

[23] L.J. Romans, Nucl. Phys. B357 (1991) 549.

[24] F. Defever, W. Troost and Z. Hasiewicz, Phys. Lett. 273B (1991) 51.

[25] K. Schoutens, A. Sevrin and P. van Nieuwenhuizen, Commun. Math. Phys. 124 (1989) 209.

[26] Z. Khviengia and E. Sezgin, Phys. Lett. 326B (1994) 243.

[27] M. Günaydin and S. Hyun, Nucl. Phys. B373 (1992) 688; M. Günaydin, Phys. Rev. D47 (1993) 3600.

[28] A. van Proeyen, Class. and Quantum Grav. 6 (1989) 1501.

[29] M. Günaydin, J. L. Petersen, A. Taormina and A. van Proeyen, Nucl. Phys. B322 (1989) 402. 
[30] S.J. Gates Jr. and S. Ketov, Phys. Rev. D52(1995)2278.

[31] B. Bina and M. Günaydin, work in preparation.

[32] A. Galperin and O. Ogievetsky, Phys. Lett. 301B (1993) 67.

[33] M. Parker, J. Math. Phys. 21 (1980) 689.

[34] J. Harvey and A. Strominger, Phys. Rev. Lett. 66 (1991) 549.

[35] T.A. Ivanova, Phys. Lett. 315B (1993) 277.

[36] M. Günaydin and H. Nicolai, Phys. Lett. 351B (1995) 169.

[37] M. Günaydin, "Conformal symmetry and duality groups of supergravity and superstring theories", talk given in Orbis Scientiae 1997 ( to appear in the proceedings of the conference).

[38] B. Julia and H. Nicolai, "Conformal internal symmetry of $2 \mathrm{~d} \sigma$-models coupled to gravity and a dilaton" , hep-th/9608082 (LPTENS 96/38; DESY 96-124).

[39] M. Günaydin, B.E.W. Nilsson, G. Sierra and P.K. Townsend, Phys. Lett. 176B (1986) 45.

[40] N. Berkovits, Nucl. Phys. B395 (1993) 77; N. Berkovits, Nucl. Phys. B420 (1994) 332 ; N. Berkovits, Nucl. Phys. B431 (1994) 258.

[41] J. de Boer and K. Skenderis, Nucl. Phys. B481 (1996) 129. 